\title{
La evolución de la movilidad escolar intergeneracional en el Perú a lo largo del siglo XX
}

L'évolution de la mobilité scolaire intergénérationnelle au Pérou au cours du XXème siècle

Evolution of Intergenerational Educational Mobility in Peru Throughout The

Twentieth Century

\section{Laure Pasquier-Doumer}

\section{OpenEdition}

Journals

Edición electrónica

URL: http://journals.openedition.org/bifea/6542

DOI: $10.4000 /$ bifea.6542

ISSN: 2076-5827

\section{Editor}

Institut Français d'Études Andines

Edición impresa

Fecha de publicación: 1 diciembre 2002

Paginación: 429-471

ISSN: 0303-7495

\section{Referencia electrónica}

Laure Pasquier-Doumer, " La evolución de la movilidad escolar intergeneracional en el Perú a lo largo del siglo XX », Bulletin de l'Institut français d'études andines [En línea], 31 (3) | 2002, Publicado el 08 diciembre 2002, consultado el 08 diciembre 2020. URL : http://journals.openedition.org/bifea/6542 ; DOI : https://doi.org/10.4000/bifea.6542

\section{(c) $(9) \odot$}

Les contenus du Bulletin de l'Institut français d'études andines sont mis à disposition selon les termes de la licence Creative Commons Attribution - Pas d'Utilisation Commerciale - Pas de Modification 4.0 International. 
Bull. Inst. fr. études andines

2002, 31 (3): 429-471

\section{LA EVOLUCIÓN DE LA MOVILIDAD ESCOLAR INTERGENERACIONAL EN EL PERÚ A LO LARGO DEL SIGLO XX}

Laure PASQUIER-DOUMER **

\section{Resumen}

El considerable desarrollo del sistema educativo escolar que caracteriza al Perú desde inicios del siglo XX parece sugerir que el acceso a la escuela se ha vuelto más igualitario. En este artículo, se busca investigar si realmente la población en general se ha beneficiado de este desarrollo de la misma manera y si este proceso ha estado acompañado de una igualación de las oportunidades escolares para todos. Para ello, se analiza la evolución de las desigualdades sociales en la escuela a lo largo del siglo XX, estudiando la evolución del vínculo entre el nivel de educación escolar de las personas y su origen cultural. Seguidamente, se examina si el debilitamiento aparente de este vínculo se explica por una mayor igualdad de oportunidades escolares o si no es más que el resultado de la prolongación generalizada de los estudios. Por último, se trata de ver qué políticas públicas podrían llevarse a cabo para aumentar la igualdad de oportunidades educativas. Es relativamente nuevo que esta problemática pueda tratarse en un país en desarrollo, gracias a la base de datos excepcional de que se dispone aquí.

Palabras claves: Desigualdad de oportunidades, movilidad escolar, exclusión, Perú, siglo XX, modelos log-lineales.

\section{L'ÉVOLUTION DE LA MOBILITÉ SCOLAIRE INTERGÉNÉRATIONNELLE AU

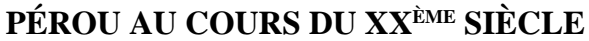

\section{Résumé}

Le développement considérable de l'enseignement scolaire qui caractérise le Pérou depuis le début du $20^{\text {ème }}$ siècle suggère que l'accès à l'éducation est devenu plus égalitaire. Dans cet article, je cherche à savoir si réellement le développement a profité à tous de la même manière et s'il s'est accompagné d'une égalisation des opportunités scolaires. Pour cela, j'analyse l'évolution des inégalités sociales devant l'école tout au long du vingtième siècle en étudiant

* Agradezco muy especialmente a José Rodríguez que me permitió tener acceso a los datos de las encuestas, sin los cuales este artículo no habría sido posible. Quiero también agradecer a Javier Herrera, Martín Benavides y Denis Cogneau por sus comentarios pertinentes, a María Polo Puelles y las otras personas del Ministerio de Trabajo que me dieron acceso a los archivos de las encuestas antiguas y a ciertas encuestas. Traducción al castellano de Lucie Kearns Vézina.

** Instituto Francés de Estudios Andinos (IFEA), Contralmirante Montero 141, casilla 181217, Lima 18 - Perú. Institut d'Études Politiques, Dial. E-mail: laurepas@ hotmail.com 
l'évolution du lien entre le niveau d'éducation des personnes et leur origine culturelle. Ensuite, j'examine si le relâchement de ce lien que l'on observe s'explique par une plus forte égalité d'opportunité scolaire ou s'il n'est que le résultat de l'allongement généralisé des études. Enfin, j'étudie quelles politiques publiques pourraient être conduites pour accroître l'égalité des chances devant l'école. L'originalité de cet article est de pouvoir aborder cette problématique pour un pays en développement grâce à la base de données exceptionnelle dont on dispose ici.

Mots clés : Inégalités d'opportunité, mobilité scolaire, exclusion, Pérou, vingtième siècle, modèles log-linéaires.

\title{
EVOLUTION OF INTERGENERATIONAL EDUCATIONAL MOBILITY IN PERU THROUGHOUT THE TWENTIETH CENTURY
}

\begin{abstract}
Since the beginning of the twentieth century, the education system in Peru has known a considerable development. This suggests that in all probabilities opportunities for study should be on a more equal basis. In the present article, I set out to discover whether all social groups have really benefited from the development of school system and whether this development created equal opportunities or not. I there for analyze the evolution of educational inequalities throughout the twentieth century by basing my observations on the evolution of the relationship between the educational level attained and the sociocultural origin of the person. I then examine whether the apparently diminishing importance of this relationship is due to the opening up of a vaster field of equal opportunities or whether the principal factor is the generalized prolongation of studies. Finally, I examine what public policies could be pursued in order to increase equal educational opportunities. This is the first time that thanks to the exceptional quality of the data at our disposal, this issues can be deals with in relationship to a developing country.
\end{abstract}

Key words: Inequalities of opportunity, educational mobility, exclusion, Peru, twentieth century, log-linear models.

En el Perú, la educación escolar se percibe desde hace tiempo como un vehículo potente de ascención social. En 1967 (Ministerio de Trabajo, 1967), en la primera encuesta representativa de la población de Lima que se haya realizado en el Perú, todos los jefes de familia reconocen la importancia de la educación "para salir adelante" (1). Aún en 1995, mientras que muchos piensan que la crisis económica y social de fines de los años 80 y principio de los años 90 ha erosionado la enorme confianza que tienen los padres en la educación, Ansión et al. (1998) ponen en evidencia que un 95\% de los padres limeños (2) desean que sus hijos alcancen un nivel escolar superior al secundario, y dos tercios que tengan un título universitario. Además, una gran mayoría de los padres creen que la educación es un medio para mejorar sus condiciones de vida (3) a pesar de la disminución evidente de la calidad de la educación y la falta de perspectivasen el mercado laboral.

(1) Todos respondieron a la pregunta "¿Cuál es la importancia de la educación para salir adelante?","muy importante" o "importante".

(2) Encuesta realizada por los autores en 1995 con 462 hogares a partir de un cuestionario y con 35 hogares en entrevista abierta. La muestra se construye a partir de la muestra del ENNIV-94 en Lima Metropolitana ( 847 hogares) y para la cual se retuvieron los hogares con hijos en edad escolar. dinero!".

(3) Casi dos tercios de los padres están de acuerdo con la afirmación: “¡A más educación, más 
En este contexto, la igualdad de acceso a la escuela es una meta prioritaria en el Perú. Primero, porque el entusiasmo hacia la educación es compartido por todos. Segundo, porque permitiría reducir las desigualdades sociales desde su raíz. En efecto, si la educación en el Perú no es una condición suficiente para alcanzar una posición social alta, es cierto que en la mayoría de los casos es una condición necesaria. Así pues, desigualdades en los niveles de educación se traducen más adelante en desigualdades socioeconómicas.

El fuerte crecimiento de los niveles de educación y de la cobertura escolar que caracteriza al Perú desde principios del siglo pasado sugiere que el acceso a la educación se ha vuelto más igualitario. ¿Pero realmente es así? ¿La expansión del sistema escolar benefició a todos de la misma manera? ¿Se acompañó de una igualación de las oportunidades escolares? (4)

El objetivo de este artículo es estudiar la evolución de las desigualdades sociales en la escuela a lo largo del siglo XX, estudiando la evolución del vínculo entre el nivel de educación de los padres y el de sus hijos. En particular, buscará analizar dicha evolución en sus dos componentes, es decir, por una parte, la prolongación de los estudios y, por otra parte, la evolución de las probabilidades relativas o la evolución de la desigualdad de oportunidades en el acceso a la escuela. Por último, evaluará las distintas políticas públicas que pueden mejorar el grado de igualdad de oportunidades ante la escuela. Esta problemática ya se trabajó en países desarrollados (Blossfeld \& Shavit, 1993; Heath \& Payne, 1999; Thélot \& Vallet, 2000; Ganzeboom \& Luijkx, 2001) pero, según tengo entendido, nunca en un país en vías de desarrollo. Se ha demostrado en varios estudios (Blossfeld \& Shavit, 1993; Erikson \& Goldthorpe, 1992) que los países desarrollados siguen un mismo tipo de evolución en términos de igualdad de oportunidades. La cuestión consiste entonces en saber si un país en desarrollo sigue este mismo proceso. Además, entender cómo se forman las desigualdades y cuáles son las posibilidades de reducirlas parece esencial en un país como el Perú, que alcanza un grado de desigualdad difícilmente soportable, fuente de inestabilidad social y política.

Se llevaron a cabo varios análisis acerca de la movilidad escolar en países en desarrollo y, en particular, en América Latina (Behrman et al., 1998; 2001; Andersen, 2001a; b; Dahan \& Gaviria, 1999; Bourguignon et al., 2001), pero ninguno sobre la evolución de la movilidad escolar y aún menos sobre la evolución de la desigualdad de oportunidades escolares, aparte de la investigación que hicieron Binder \& Woodruff (2002) sobre México. Sin embargo, los autores de este estudio disponen de los datos de una sola encuesta, lo que constituye un periodo de estudio bastante corto.

Para analizar la evolución de la desigualdad de oportunidades en la escuela, se dispone aquí de una base de datos excepcional, poco común en un país en desarrollo, que reúne varias encuestas realizadas entre 1974 y 2001. Una primera parte presentará los datos. Se puede por fin, gracias a esta base de datos, realizar un primer estudio de los mecanismos de la movilidad social sobre un largo periodo en un país en desarrollo.

(4) El concepto de igualdad de oportunidades utilizado aquí es el de Roemer (1998). Se definirá en el artículo (véase p. 17-18). 
El objeto de la segunda parte es describir la evolución del sistema escolar peruano en el siglo XX. Contestará las siguientes preguntas: ¿Cuál es la amplitud de la expansión de la cobertura educativa? ¿Quién la ha aprovechado más? ¿Quién ha sido excluido?

La tercera parte analizará la evolución del vínculo entre el éxito escolar de una persona y su origen cultural, habiendo definido previamente una medida de la movilidad escolar y estudiado la evolución de la movilidad escolar a lo largo del tiempo para, finalmente, desagregar este vínculo con el fin de analizar la evolución de la desigualdad de oportunidades escolares. La cuarta parte tratará de los factores que bloquean el aumento de la igualdad de oportunidades en la educación y de las distintas políticas que pueden llevarse a cabo para limitar la influencia de estos factores. La última parte presentará las conclusiones.

\section{PRESENTACIÓN DE LOS DATOS}

Este análisis utiliza cinco encuestas realizadas en 1974, 1985-1986, 1990, 1996 y 2001. Todas contienen un módulo sobre los padres de la persona entrevistada, lo que incluye la educación de los padres y, con excepción de la encuesta 2001, la ocupación de los padres. Las encuestas de 1974, 1990 y 1996 son encuestas de empleo realizadas por el Ministerio de Trabajo peruano. Las de 1985-1986 y 2001 son encuestas sobre el nivel de vida, la primera desarrollada según la metodología LSMS ("Living Standard Measurement Survey") por el Banco Mundial y el Instituto Nacional de Estadística y Informática del Perú (INEI), la segunda por el INEI. Sus características se encuentran sintetizadas en el cuadro 1.

Cuadro 1 - Presentación de las encuestas.

\begin{tabular}{|c|c|c|c|c|c|c|c|}
\hline Años & Meses & Nombre & Cobertura & $\begin{array}{r}\text { Tama } \\
\text { mı } \\
\text { Hogares }\end{array}$ & $\begin{array}{l}\text { de la } \\
\text { tra } \\
\text { dividuos }\end{array}$ & $\begin{array}{l}\text { Restricción } \\
\text { del campo }\end{array}$ & $\begin{array}{c}\text { Notas sobre el } \\
\text { modulo:caract. } \\
\text { de los padres }\end{array}$ \\
\hline 1974 & feb.-abr. & $\begin{array}{l}\text { Encuesta Reg. Urb. } \\
\text { de Mano de Obra }\end{array}$ & $\begin{array}{c}\text { Ciudades de } \\
\text { más de } 12500 \\
\text { hab. }\end{array}$ & 5460 & 15655 & $\begin{array}{l}\text { Los de } 14 \\
\text { años y más }\end{array}$ & $\begin{array}{c}\text { No existe } \\
\text { información sobre } \\
\text { la madre }\end{array}$ \\
\hline $1985 / 86$ & $\begin{array}{l}\text { jul. } 85- \\
\text { jul. } 86\end{array}$ & $\begin{array}{l}\text { Encuesta Nac. de Ho- } \\
\text { gares sobre Medición } \\
\text { de Niveles de Vida }\end{array}$ & Nacional & 5108 & 26323 & - & \\
\hline 1990 & jul.-agto & $\begin{array}{c}\text { Encuesta de Niveles } \\
\text { de Empleo en Lima } \\
\text { Metropolitana }\end{array}$ & Lima Callao & 1734 & 5738 & $\begin{array}{l}\text { Los de } 14 \\
\text { años y más }\end{array}$ & \\
\hline 1996 & III Trim. & $\begin{array}{c}\text { Encuesta espeziali- } \\
\text { da de Niveles de } \\
\text { Empleo }\end{array}$ & $\begin{array}{l}\text { Ciudades de más } \\
\text { de } 12500 \text { hab. }\end{array}$ & 12546 & 41998 & $\begin{array}{l}\text { Los de } 14 \\
\text { años y más }\end{array}$ & \\
\hline 2001 & IV Trim. & $\begin{array}{l}\text { Encuesta nacional de } \\
\text { Hogares sobre condi- } \\
\text { ciones de vida y pobreza }\end{array}$ & Nacional & 18179 & 70249 & - & $\begin{array}{c}\text { No está la ocupación de } \\
\text { los padres. Sólo info. sobre } \\
\text { los padres del jefe del hogar y } \\
\text { de su cónyugue }\end{array}$ \\
\hline
\end{tabular}


En estas encuestas, la información sobre los estudios realizados es de tipo retrospectiva, lo que permite conocer el nivel de estudios alcanzado por la persona. En la mayoría de los estudios sobre la movilidad escolar en los países en desarrollo, los autores no disponen de encuestas que permitan conocer el grado de educación alcanzado, puesto que las personas estudiadas son niños que todavía viven en el hogar de sus padres y que, a menudo, no han terminado sus estudios. Por ejemplo, Behrman et al. [1998; 2001 (5)] estudian la movilidad escolar preguntándose cómo el retraso escolar de un niño que vive con sus padres depende de las características de los padres, en particular, su educación. Andersen (2001a) utiliza también el retraso escolar como variable explicativa que regresa sobre dos variables de origen: el ingreso del hogar por adulto, el nivel de educación del padre más educado y sobre variables individuales. Dahan \& Gaviria (1999) adoptan un planteamiento un poco diferente, pero basado siempre en el retraso escolar de los niños. Su objetivo consiste en determinar si, entre dos hermanos, las diferencias de retraso escolar son las mismas que las de dos individuos elegidos de modo aleatorio. Si las diferencias entre hermanos son menores, es que la influencia de la familia desempeña un papel importante en el éxito escolar y que, por lo tanto, la movilidad escolar intergeneracional es baja. La crítica que se puede dirigir a este modelo es que, por una parte, considera sólo los hogares que tienen al menos dos hijos — lo que genera un sesgo vinculado a la fertilidad diferencial- y, por otra parte, supone que el trato a los hijos es el mismo en el hogar, hipótesis difícilmente aceptable, sobre todo en países en desarrollo. El problema con enfocar el nivel escolar final desde el retraso escolar en un momento dado crea un sesgo de selección. De hecho, se puede pensar que los hijos poco instruidos permanecen menos tiempo en su familia y empiezan antes que los demás su vida independiente. Además, considerar el retraso escolar excluye del análisis todos los hijos que no están escolarizados. Por último, la relación entre el retraso escolar y el nivel finalmente alcanzado no es evidente: un hijo puede conocer dificultades al principio de su escolaridad y superarlas luego. Y, al contrario, un hijo puede no tener retraso escolar y, sin embargo, tener un bajo nivel escolar si, por ejemplo, los padres no tienen los recursos suficientes para seguir financiando la escolaridad de su hijo y lo obligan a salir prematuramente del sistema escolar. Disponer de datos retrospectivos permite evitar estos inconvenientes. A pesar de ello, se pierde mucha información sobre el entorno familiar de la persona en el momento en que realizó su escolaridad. Otro inconveniente de los datos retrospectivos es que dependen de las variaciones de la memoria. Pero aquí, el problema no es muy grave, puesto que se trata de una comparación en el tiempo y que, a priori, el sesgo es el mismo en cada una de las encuestas.

En cada una de las encuestas se han retenido a las personas mayores de 23 años y menores de 70 años. El límite inferior es para asegurarse de que todos los encuestados terminaron sus estudios y el límite superior, para evitar un sesgo vinculado a la mortalidad diferencial (6).

(5) La primera parte de este artículo analiza también, para algunos países, la movilidad escolar desde el nivel de educación alcanzado por la persona y el nivel de educación alcanzado por el padre, ya que los autores disponen de datos retrospectivos para estos países.

(6) Si se supone, por ejemplo, que una persona cuyo nivel escolar es elevado tiene un nivel de vida superior y una esperanza de vida mayor a los de una persona con bajo nivel escolar, pues, para la generación de mayor edad, estaríamos sobrestimando la proporción de personas con nivel escolar elevado. 
A partir de estas encuestas, se pudieron constituir siete generaciones decenales. La de mayor edad nació a principios del siglo XX, la más reciente en los años setenta. Se pudieron elaborar además tres zonas de análisis: Lima, las otras ciudades — ciudades de más de 12500 habitantes fuera de Lima - y el Perú rural. Estas tres zonas de análisis proporcionan una visión global del Perú. No se pueden sin embargo mezclar, ya que la muestra no sería más representativa, las coberturas de las encuestas siendo muy diferentes. Por ello, se tuvo que analizar simultáneamente cada una de las zonas.

La ventaja que brindan tantas encuestas, además de alargar el periodo de análisis, es de tener una cantidad suficientemente representativa de individuos por generación y por zona de estudio. Se acumularon, en efecto, para conformar las generaciones, las muestras de las distintas encuestas comunes a cada generación (7). La base de datos representativa de Lima agrupa las muestras que se refieren a Lima de todas las encuestas. La base representativa de las otras ciudades está conformada por las muestras de todas las encuestas, excepto la de 1990 — que sólo cubre Lima-. Y finalmente, la base representativa del Perú rural es la base con la menor cantidad de representantes, puesto que solamente las encuestas de 1985-1986 y 2001 tienen una cobertura rural. Los cuadros 2, 3 y 4 presentan la cantidad de representantes de cada zona de análisis en función de las generaciones y encuestas.

Para poder agregar las encuestas, fue necesario aplicar a los datos una ponderación que tenga en cuenta las diferencias de los tipos de sondeo entre las encuestas. Para cada zona de análisis, se extrapolaron las observaciones de cada encuesta, gracias a su factor de expansión y luego, con una simple regla de tres, ajusté el número de individuos extrapolado de cada encuesta al número real de individuos entrevistados. Así, en cada zona de análisis, cada observación $i$ de la encuesta $e$ fue ponderada por el peso $p_{i}^{e}$ siguiente:

$$
p_{i}^{e}=\frac{f_{i}^{e} \times \sum_{e} n_{e}}{\sum_{e} \sum_{i}^{e} f_{i}^{e}}
$$

en donde $f_{i}^{e}$ es el factor de expansión de la observación $i$ de la encuesta $e$ y $n_{e}$ es el número de observaciones de la encuesta $e$ para la zona estudiada, con $e=1974,1985$, 1990, 1996, 2001 para Lima, $e=1974,1985,1996,2001$ para las otras ciudades y $e=1985,2001$ para el medio rural.

(7) Para comprobar la coherencia de las encuestas entre ellas, se evaluó con una prueba de Kolmogorov-Smirnov si las distribuciones de los niveles de educación son estables entre las diferentes encuestas para cada generación. Es decir, se evaluó si la diferencia de las distribuciones de los años de escolaridad de dos encuestas para una misma generación era nula. La variable de los años de escolaridad realizados por la persona entrevistada se multiplicó por su ponderación para tener en cuenta los pesos en la prueba. Todas las pruebas confirman que no hay diferencia significativa entre las distribuciones de cada encuesta. 


\section{Cuadro 2 - Distribución de la muestra de las encuestas en función de las generaciones que viven en el momento de la investigación en Lima.}

\begin{tabular}{|c|c|c|c|c|c|c|}
\hline Generaciones & ERUMO 1974 & ENNIV 1985/86 & $\begin{array}{l}\text { Encuestas } \\
\text { ENAHO } 90\end{array}$ & ENAHO 96 & ENAHO 2001 & $\begin{array}{l}\text { Número total } \\
\text { de individuos }\end{array}$ \\
\hline 1905-1912 & $\begin{array}{c}\text { 62-69 años } \\
(211)\end{array}$ & & & & & 211 \\
\hline 1913-1922 & $\begin{array}{c}\text { 52-61 años } \\
\text { (450) }\end{array}$ & $\begin{array}{c}\text { 63-69 años } \\
(184)\end{array}$ & & & & 634 \\
\hline 1923-1932 & $\begin{array}{l}\text { 42-51 años } \\
\text { (852) }\end{array}$ & $\begin{array}{c}\text { 53-62 años } \\
\text { (415) }\end{array}$ & $\begin{array}{c}\text { 58-69 años } \\
\text { (389) }\end{array}$ & $\begin{array}{c}\text { 64-69 años } \\
\text { (246) }\end{array}$ & & 1902 \\
\hline 1933-1942 & $\begin{array}{c}\text { 32-41 años } \\
\text { (1324) }\end{array}$ & $\begin{array}{l}\text { 43-52 años } \\
\text { (590) }\end{array}$ & $\begin{array}{c}\text { 48-57 años } \\
\text { (574) }\end{array}$ & $\begin{array}{c}\text { 54-63 años } \\
\text { (570) }\end{array}$ & $\begin{array}{c}\text { 59-69 años } \\
\text { (559) }\end{array}$ & 3617 \\
\hline 1943-1952 & $\begin{array}{c}\text { 23-31 años } \\
\text { (1651) }\end{array}$ & $\begin{array}{c}\text { 33-42 años } \\
\text { (839) }\end{array}$ & $\begin{array}{c}\text { 38-47 años } \\
\text { (834) }\end{array}$ & $\begin{array}{c}\text { 44-53 años } \\
\text { (973) }\end{array}$ & $\begin{array}{c}\text { 49-58 años } \\
\text { (805) }\end{array}$ & 5102 \\
\hline 1953-1962 & & $\begin{array}{c}\text { 23-32 años } \\
\text { (1278) }\end{array}$ & $\begin{array}{l}\text { 27-37 años } \\
\text { (1205) }\end{array}$ & $\begin{array}{c}\text { 34-43 años } \\
\text { (1277) }\end{array}$ & $\begin{array}{c}\text { 39-48 años } \\
\text { (1083) }\end{array}$ & 4843 \\
\hline 1963-1978 & & & $\begin{array}{c}\text { 23-26 años } \\
\text { (788) }\end{array}$ & $\begin{array}{c}\text { 23-33 años } \\
(1758)\end{array}$ & $\begin{array}{c}\text { 23-38 años } \\
\text { (1365) }\end{array}$ & 3911 \\
\hline Conjunto & 4488 & 3306 & 3790 & 4824 & 3812 & 20220 \\
\hline
\end{tabular}

Lectura: Las edades indicadas son las edades en el momento de la investigación. El número entre paréntesis representa el número de individuos de la generación para cada encuesta.

Campo: Individuos que viven en Lima en el momento de la encuesta, que tienen entre 23 y 69 años. Por el ENAHO 2001, se refiere sólo a los jefes de familia y sus cónyuges.

Cuadro 3 - Distribución de la muestra de las investigaciones en función de las generaciones que viven en el momento de la investigación en zonas urbanas fuera de Lima (otras ciudades).

\begin{tabular}{|c|c|c|c|c|c|}
\hline \multirow[b]{2}{*}{ Generaciones } & \multicolumn{4}{|c|}{ Encuestas } & \\
\hline & ERUMO 1974 & ENNIV 1985/86 & ENAHO 96 & ENAHO 2001 & \\
\hline 1905-1912 & $\begin{array}{c}62-69 \text { años } \\
(340)\end{array}$ & & & & 340 \\
\hline 1913-1922 & $\begin{array}{c}\text { 52-61 años } \\
\text { (639) }\end{array}$ & $\begin{array}{c}\text { 63-69 años } \\
(188)\end{array}$ & & & 827 \\
\hline 1923-1932 & $\begin{array}{c}\text { 42-51 años } \\
\text { (1220) }\end{array}$ & $\begin{array}{c}\text { 53-62 años } \\
(406)\end{array}$ & $\begin{array}{c}\text { 64-69 años } \\
\text { (984) }\end{array}$ & & 2610 \\
\hline 1933-1942 & $\begin{array}{c}\text { 32-41 años } \\
(1618)\end{array}$ & $\begin{array}{c}\text { 43-52 años } \\
\text { (621) }\end{array}$ & $\begin{array}{c}\text { 54-63 años } \\
\text { (2692) }\end{array}$ & $\begin{array}{c}\text { 59-69 años } \\
(1471)\end{array}$ & 6402 \\
\hline 1943-1952 & $\begin{array}{c}\text { 23-31 años } \\
(1755)\end{array}$ & $\begin{array}{c}\text { 33-42 años } \\
\text { (809) }\end{array}$ & $\begin{array}{c}\text { 44-53 años } \\
\text { (4335) }\end{array}$ & $\begin{array}{c}\text { 49-58 años } \\
\text { (2129) }\end{array}$ & 9028 \\
\hline 1953-1962 & & $\begin{array}{c}\text { 23-32 años } \\
(1165)\end{array}$ & $\begin{array}{c}\text { 34-43 años } \\
(6340)\end{array}$ & $\begin{array}{c}\text { 39-48 años } \\
\text { (3334) }\end{array}$ & 10839 \\
\hline 1963-1978 & & & $\begin{array}{c}\text { 23-33 años } \\
(8717)\end{array}$ & $\begin{array}{c}\text { 23-38 años } \\
\text { (4518) }\end{array}$ & 13235 \\
\hline Conjunto & 5572 & 3189 & 23068 & 11452 & 43281 \\
\hline
\end{tabular}

Lectura: Las edades indicadas son las edades en el momento de la investigación. El número entre paréntesis representa el número de individuos de la generación para cada encuesta. 
Campo: Individuos que viven en zonas urbanas fuera de Lima en el momento de la encuesta, que tienen entre 23 y 69 años. Por el ENAHO 2001, se refiere sólo a los jefes de familia y sus cónyuges.

\section{Cuadro 4 - Distribución de la muestra de la investigación en función de las generaciones que viven en el momento de la investigación en zonas rurales.}

\begin{tabular}{|c|c|c|c|}
\hline \multicolumn{4}{|c|}{ Encuestas } \\
\hline Generaciones & ENNIV 1985/86 & ENAHO 2001 & \\
\hline 1913-1922 & $\begin{array}{c}\text { 63-69 años } \\
\text { (344) }\end{array}$ & & 344 \\
\hline 1923-1932 & $\begin{array}{c}\text { 53-62 años } \\
\text { (733) }\end{array}$ & & 733 \\
\hline 1933-1942 & $\begin{array}{c}\text { 43-52 años } \\
\text { (1022) }\end{array}$ & $\begin{array}{c}\text { 59-69 años } \\
(1440)\end{array}$ & 2462 \\
\hline 1943-1952 & $\begin{array}{c}\text { 33-42 años } \\
(1186)\end{array}$ & $\begin{array}{l}\text { 49-58 años } \\
\text { (1763) }\end{array}$ & 2949 \\
\hline 1953-1962 & $\begin{array}{c}\text { 23-32 años } \\
\text { (1331) }\end{array}$ & $\begin{array}{l}\text { 39-48 años } \\
\text { (2403) }\end{array}$ & 3734 \\
\hline 1963-1978 & & $\begin{array}{c}\text { 23-38 años } \\
\text { (4174) }\end{array}$ & 4174 \\
\hline Conjunto & 4616 & 9780 & 14396 \\
\hline
\end{tabular}

Lectura: Las edades indicadas son las edades en el momento de la investigación. El número entre paréntesis representa el número de individuos de la generación para cada encuesta.

Campo: Individuos que viven en zonas rurales en el momento de la encuesta, que tienen entre 23 y 69 años. Por el ENAHO 2001, se refiere sólo a los jefes de familia y sus cónyuges.

\section{EVOLUCIÓN DE LA COBERTURA ESCOLAR A LO LARGO DEL SIGLO}

Uno de los fenómenos más significativos para la sociedad peruana del siglo XX es la expansión considerable de la educación. Se tradujo, por una parte, en un aumento del tiempo de estudios y, por otra parte, en un acceso mucho más generalizado a la escuela.

\section{1. Prolongación general de los estudios y aumento de la distancia entre lo urbano y lo rural}

En Lima, la cantidad promedio de años de escolaridad se duplicó entre la generación nacida a principios del siglo y la nacida en los años setenta (Fig. 1). En las otras ciudades, se multiplicó por más tres, pasando de 3 años a 9,5 años, alcanzando casi el nivel de Lima. En el medio rural, sin embargo, la prolongación de los estudios es 
reducida. En sesenta años, el nivel escolar se incrementó por un promedio de solamente 3 años. La diferencia entre la duración promedio de los estudios en Lima y la duración promedio en el medio rural se mantuvo casi constante en el tiempo, mientras que la misma entre las otras ciudades y el medio rural se acentuó bastante. Si bien las diferencias entre Lima y las otras ciudades en cuanto a la duración de los estudios casi desaparecieron durante el siglo, se acentuaron bastante entre el medio rural y el medio urbano.

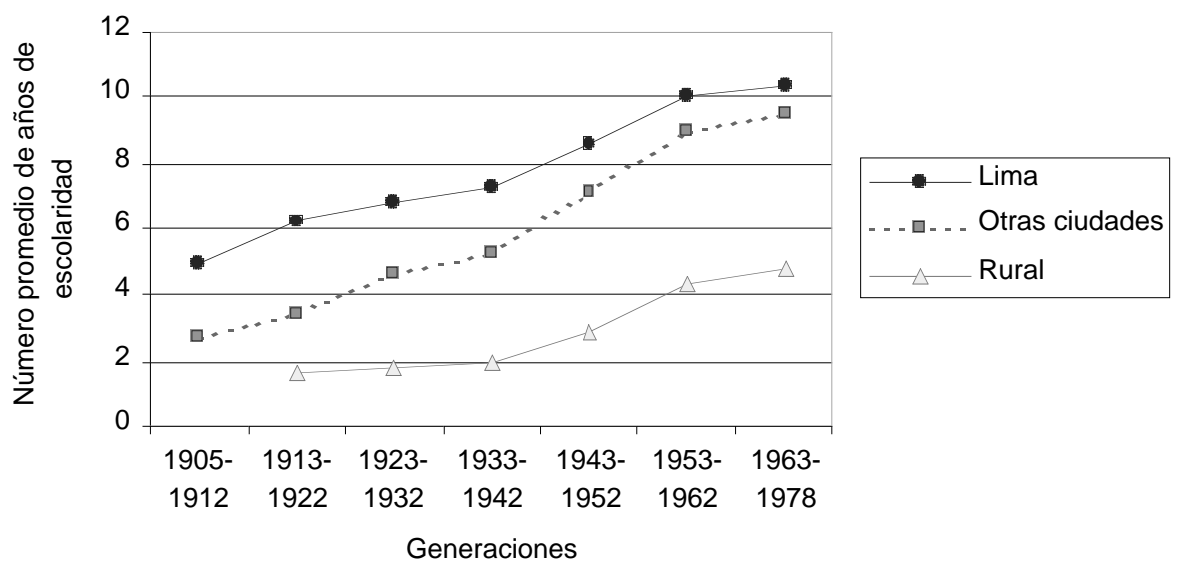

Fuentes: encuestas 1974, 1985-1986, 1990, 1996, 2001.

Fig. 1 - Evolución de la escolaridad en el medio urbano y el medio rural (8).

\section{2. Democratización de la enseñanza primaria, pero exclusión del medio rural a la apertura de la enseñanza superior}

El desarrollo de la enseñanza primaria es la característica quizás más destacada de la evolución del sistema educativo en el Perú (Fig. 2), pero también la apertura de la enseñanza superior (Fig. 3). En las ciudades, la enseñanza primaria se ha vuelto casi universal y la diferencia entre Lima y las otras ciudades se ha suprimido. Si bien en el medio rural la enseñanza primaria no es universal, su desarrollo es muy rápido, sobre todo durante el periodo en que se educa a la generación nacida entre 1933 y 1942. Su índice de crecimiento en ese momento es bastante superior al que se observa en medio urbano. Sin embargo, este rápido crecimiento no basta para nivelar la diferencia entre el medio urbano y el medio rural, puesto que, para la última generación, la diferencia

(8) La diferencia entre la curva de evolución de la escolaridad en Lima y la de las otras ciudades es estadísticamente significativa, así como la diferencia entre la curva de evolución de la escolaridad en las otras ciudades y la del medio rural. Eso se comprobó gracias a pruebas de dominio de tipo Kolmogorov-Smirnov aplicadas a cada generación y que consisten en evaluar si, para una generación dada, la función de distribución de los años de escolaridad de una zona de análisis domina estocasticamente la función de distribución de los años de escolaridad de otra zona geográfica. Esta comprobación permite afirmar de manera más segura los resultados observados a partir del gráfico 1. 
sigue siendo considerable, de más de 30 puntos. Es difícil explicar por qué se ve excluida una parte tan importante de la población infantil en el medio rural de este recurso al cual todos deberían tener acceso. ¿Es esto imputable a una insuficiente oferta de educación primaria - la falta de escuelas, los profesores poco capacitados y mal pagados promueven el ausentismo y el fracaso escolar-? o ¿el costo para los padres de enviar a sus hijos a la escuela es demasiado elevado? Lo cierto es que los que viven en el medio rural sufren de una desventaja en lo que se refiere al acceso a la escuela primaria. Sin embargo, es necesario relativizar esta observación, destacando que muchos de los niños nacidos en el medio rural han migrado hacia la ciudad, en particular, para conseguir educación secundaria o superior. Han permanecido los que tienen el nivel de educación más bajo. En todo caso, la proporción de los que no completaron la escuela primaria sigue siendo muy importante. Cabe tener en cuenta que el fuerte crecimiento de la tasa de participación en la escuela primaria, que empieza en los años en que se educa la generación nacida entre 1933 y 1942, sucede al mismo tiempo que un importante aumento en el presupuesto nacional para la educación.

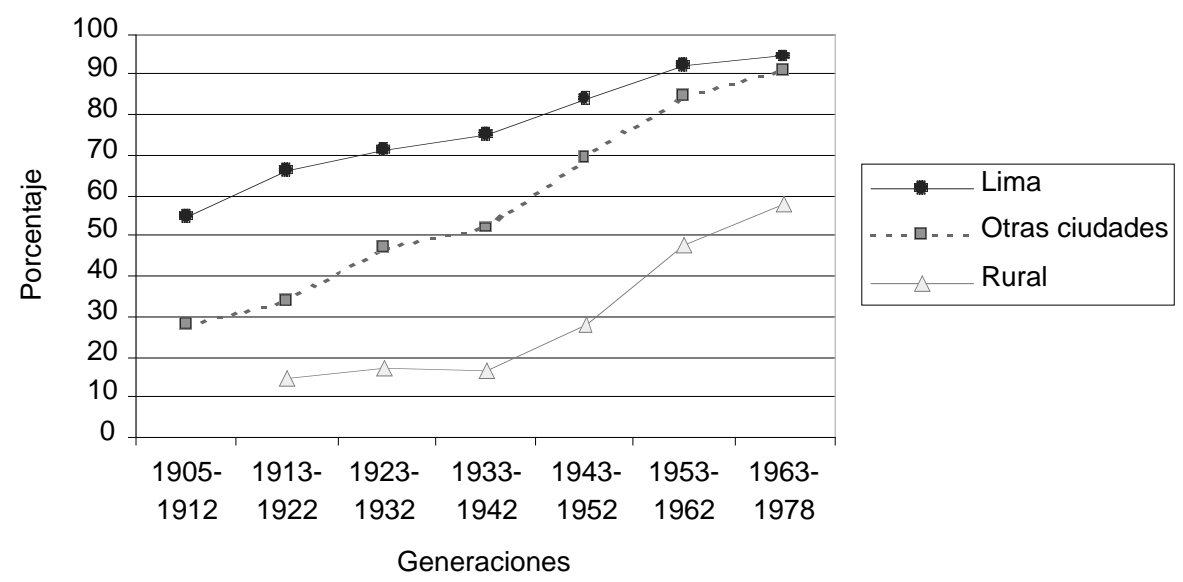

Fuentes: encuestas 1974, 1985-1986, 1990, 1996, 2001.

\section{Fig. 2 - Evolución del porcentaje de la población total habiendo terminado sus estudios primarios (urbano y rural).}

A inicios del siglo XX, la enseñanza superior era accesible sólo a una elite muy reducida. Su expansión se llevó a cabo en las ciudades de manera continua, con un alto índice de crecimiento, del orden de un $20 \%$ de la población, sobre todo en zonas urbanas fuera de Lima. En cambio, la cobertura de la enseñanza superior casi no ha evolucionado en el medio rural, quedándose a un nivel muy bajo, casi nulo. Este último resultado se explica fácilmente por la muy débil oferta de empleos calificados en el medio rural. Puesto que para seguir estudios superiores es necesario migrar a la ciudad, solamente las personas calificadas que tienen una oportunidad de ejercer su profesión dejan la ciudad en donde existe más demanda de trabajo calificado y regresan al medio rural para 
ejercer profesiones como las de médico, profesor o personal de la administración pública. Así pues, y es lo que se observa en la figura 3, la proporción de los que han conseguido una educación superior corresponde perfectamente en el medio rural a la proporción de los empleos calificados (9) en el mercado laboral rural. La correlación entre la demanda de trabajo calificado y la proporción de personas calificadas es también muy fuerte en zonas urbanas, aunque en menor medida. Ambas conocen la misma evolución: un fuerte crecimiento hasta los años durante los cuales la generación nacida entre 1953-1962 tiene la edad de conseguir una educación superior y una fuerte reducción durante los años de estudios de la última generación. Así, todo indica que la demanda de educación superior está muy vinculada a la situación del mercado laboral. Sin embargo, en medio urbano la proporción de las personas que han obtenido una educación superior supera la de los empleos calificados — para Lima, desde los años de estudios de la primera generación y para las otras ciudades, a partir de los años de estudios de la generación nacida entre 1933 y 1942 - y la diferencia se incrementa con el tiempo. Para la última generación, la diferencia es de casi 10 puntos.
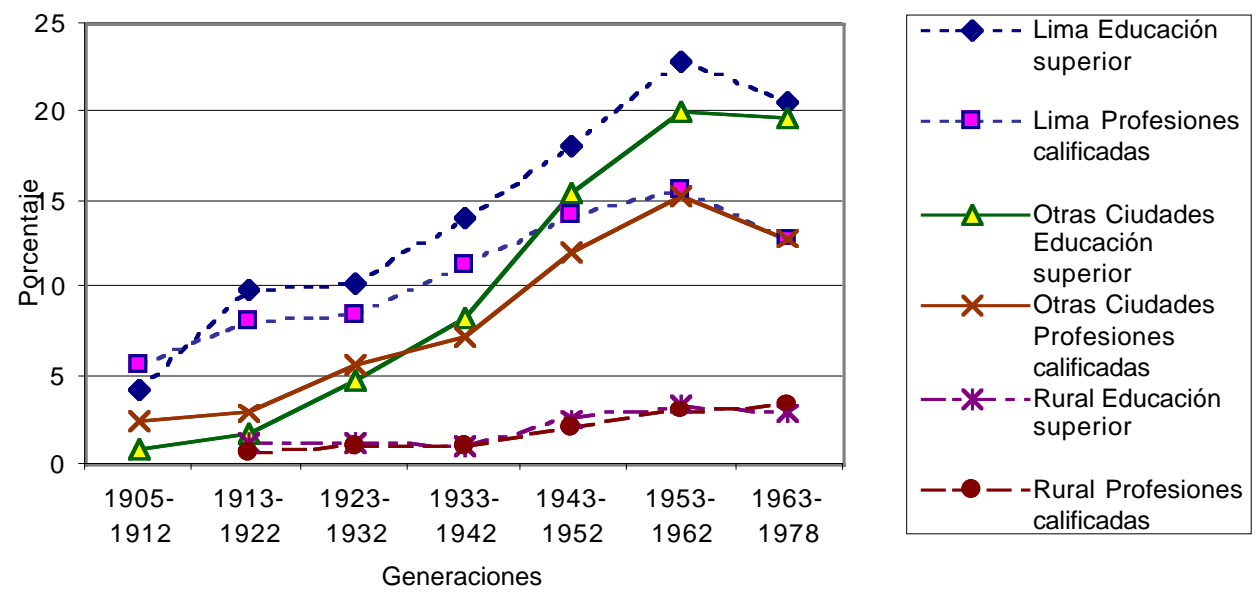

Fuentes: encuestas 1974, 1985-1986, 1990, 1996, 2001

Fig. 3 - Evolución de la proporción de la población que acaba un ciclo superior comparada con la evolución de la proporción de las profesiones calificadas (urbano y rural).

(9) Este resultado contradice el dado por el modelo de Boudon (1973) para las sociedades industrializadas liberales que afirma que el incremento de la demanda de educación se concibe como "la consecuencia de factores principalmente endógenos — cuando la demanda de una categoría social aumenta, los otros deben aumentar la suya so pena de ver sus esperanzas sociales reducidas-, aunque factores exógenos —incidencia de los cambios económicos, tecnológicos, etc.— desempeñan también un papel en la explicación de los cambios en la demanda de educación. Por lo tanto, los cambios de la estructura educativa no tienen ninguna razón de ser congruentes con los cambios de la estructura socio-profesional" (p. 306). 
La escasa prolongación de los estudios que se observa en el medio rural, comparándola con la del medio urbano, se explica en parte por el bajo porcentaje de los que han realizado estudios superiores y que se mantuvieron en el medio rural, dado que la movilidad escolar de los habitantes rurales se acompaña en general de una movilidad geográfica.

\section{3. La brecha entre el nivel escolar de las niñas y el de los niños se ha suprimido, excepto en el medio rural}

Antes de la generación nacida en 1943-1952, el nivel de educación promedio de los niños superaba de cerca de dos años el de las niñas, tanto en el medio urbano como en el rural (Fig. 4). A partir de esa generación, se empieza un movimiento de igualación en las ciudades y de diferenciación entre los géneros en el acceso a la escolaridad en el medio rural. En Lima, en veinte años, la participación de las niñas se ha vuelto casi la misma que la de los niños. En las otras ciudades, una ligera diferencia persiste, pero es muy débil. En el medio rural por el contrario, la diferencia se ha acentuado con el tiempo, aunque se reduce ligeramente en el caso de la última generación. La diferencia es todavía de dos años para la última generación. Así pues, si en las ciudades los padres casi no favorecen más a los niños en desmedro de sus hermanas, este comportamiento discriminatorio en las familias está aún presente en el medio rural.

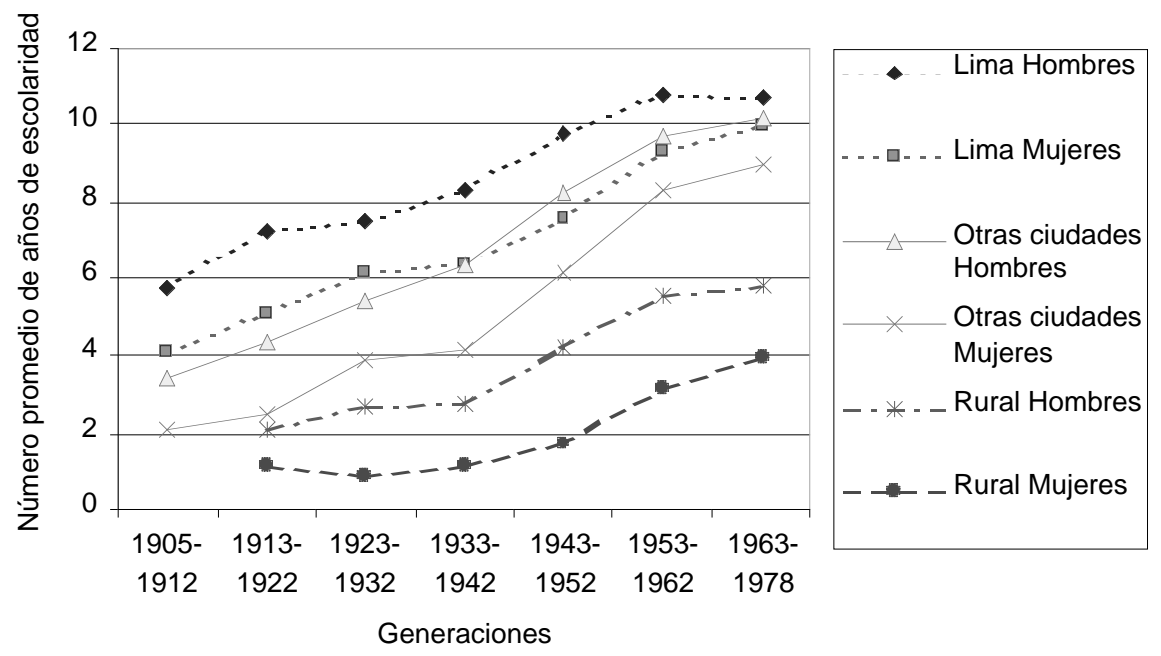

Fuentes: encuestas 1974, 1985/1986, 1990, 1996, 2001

Fig 4 - Evolución de la escolaridad en zona urbana y en zona rural según el sexo (10).

(10) De la misma forma que para la figura 1 (véase nota 7), se comprobó, gracias a pruebas de Kolmogorv-Smirnov aplicadas a cada generación, que las diferencias entre las funciones de distribución de los años de escolaridad — entre Lima Hombres y Lima Mujeres, Lima Mujeres y Otras ciudades Hombres, Otras ciudades Hombres y Otras ciudades Mujeres, ... - son significativamente diferentes. 
Para entender mejor dónde ocurre la discriminación entre los géneros en el medio rural, se observaron las diferencias de probabilidad de superar las siguientes transiciones: la transición entre sin instrucción y el comienzo de la enseñanza primaria, entre la enseñanza primaria incompleta y la enseñanza primaria completa $\mathrm{y}$, finalmente, entre la enseñanza primaria completa y la secundaria. El cuadro 1 presenta estas probabilidades. Hasta la última generación, la discriminación entre niñas y niños reside principalmente en el hecho de que se envía a las niñas mucho menos frecuentemente a la escuela que a los niños. Para la generación nacida entre 1943 y 1952 por ejemplo, un $30 \%$ de las niñas no van a la escuela mientras que esta proporción es de sólo 6\% para los niños. Sin embargo, a partir de la generación nacida entre 1933 y 1942, cuando la escuela se abre masivamente a las niñas, la discriminación se ubica en el momento de decidir si permitir o no a los hijos finalizar sus estudios primarios. Para esta generación, mientras que uno de cada dos niños comienza y termina el ciclo primario, solamente una de cada tres niñas lo termina.

\section{Cuadro 5 - Probabilidad de transición en el medio rural.}

\begin{tabular}{|c|c|c|c|c|c|c|c|}
\hline Transiciones & Generaciones & 1913-1922 & 1923-1932 & 1933-1942 & 1943-1952 & $1953-1962$ & $1963-1978$ \\
\hline \multicolumn{8}{|c|}{ Sin instrucción a primaria } \\
\hline & Hombres & 57.74 & 71.64 & 77.98 & 85.93 & 93.66 & 97.68 \\
\hline & Mujeres & 27.06 & 24.52 & 34.32 & 47.94 & 70.5 & 86.44 \\
\hline & Diferencia $(\mathrm{H}-\mathrm{F})$ & 30.68 & 47.12 & 43.66 & 37.99 & 23.16 & 11.24 \\
\hline \multicolumn{8}{|c|}{ Primaria incompleta a primaria completa } \\
\hline & Hombres & 29.66 & 31.67 & 30.82 & 48.01 & 64.69 & 71.28 \\
\hline & Mujeres & 39.09 & 28.5 & 23.98 & 30.85 & 44.4 & 55.63 \\
\hline & Diferencia $(\mathrm{H}-\mathrm{F})$ & -9.43 & 3.17 & 6.84 & 17.16 & 20.29 & 15.65 \\
\hline \multicolumn{8}{|c|}{ Primaria completa a secundaria } \\
\hline & Hombres & 20.6 & 29.26 & 3.65 & 48.78 & 55.66 & 58.86 \\
\hline & Mujeres & 36.86 & 34.04 & 1.73 & 41.04 & 46.57 & 48.8 \\
\hline & Diferencia $(\mathrm{H}-\mathrm{F})$ & -16.26 & -4.78 & 1.92 & 7.74 & 9.09 & 10.06 \\
\hline
\end{tabular}

Fuentes: encuestas 1985-1986, 2001.

Lectura: La probabilidad que tiene un niño nacido entre 1913 y 1922, de nivel escolar primario incompleto de alcanzar un nivel primario completo es de 29,66.

\section{4. La discriminación étnica se ha reducido, pero sigue siendo fuerte en los niveles de enseñanza más altos}

Herrera (2002) pone en evidencia que, en el Perú, el factor étnico es un criterio de discriminación entre los pobres y los demás. ¿Se discrimina contra las personas también según un criterio étnico en su acceso a la educación? o, en otras palabras, ¿las personas indígenas tienen las mismas oportunidades de educarse que las personas no indígenas? Si la respuesta a la última pregunta es no, ¿cómo la diferencia de acceso a la educación entre indígenas y no indígenas ha evolucionado? El objetivo de esta subsección consiste en aportar luces sobre estos aspectos, complementando así el primer análisis efectuado por Herrera (2002).

La discriminación en el acceso a la educación, si existe, no es directa como puede serlo en el mercado laboral donde se niega a una persona indígena un trabajo o un sueldo 
adecuado por el solo hecho de ser indígena, aunque tenga todas las calificaciones requeridas. En el ámbito de la educación, la discriminación se establece más bien a partir de características intrínsecas vinculadas al hecho de ser indígena: la lengua materna no es la lengua de la escuela (11), los indígenas tienen padres en promedio menos escolarizados que los no indígenas, son nacidos en hogares más pobres...

La única encuesta que contenga datos sobre la etnia de la persona entrevistada es la investigación ENAHO del 2001. Por ello, el periodo de estudio se limita a las generaciones nacidas entre 1933 y 1972 (12). Esta encuesta establece un criterio "objetivo" (13) para definir si la persona es indígena o no. Uno es indígena, en una definición limitada, cuando tiene por lengua materna una lengua vernácula (quechua, aymara o lengua de la selva) y, en una definición más amplia, cuando uno de sus padres o abuelos tiene como lengua materna una lengua vernácula. El cuadro 6 presenta la evolución del porcentaje de la población que se define como indígena en función de la definición de "indígena" establecida.

\section{Cuadro 6 - Evolución de la proporción de la población indígena en la población total según la definición establecida.}

\begin{tabular}{|c|c|c|c|c|c|}
\hline $\begin{array}{ll} & \text { Generaciones } \\
\text { Definición de una persona indígena }\end{array}$ & $1932-42$ & 1943-52 & $1953-62$ & $1963-72$ & Conjunto \\
\hline Persona de lengua materna vernácula & 32.08 & 32.15 & 28.04 & 27.19 & 29.02 \\
\hline $\begin{array}{l}\text { Uno de los padres o abuelos de la persona es de } \\
\text { lengua materna vernácula }\end{array}$ & 40.44 & 43.1 & 41.56 & 43.29 & 42.57 \\
\hline
\end{tabular}

Fuente: encuesta ENAHO 2001.

La figura 5 presenta, para cada generación, el porcentaje de los que entraron en la primaria, la secundaria y la educación superior — que la hayan completado o nosegún que la persona tenga por lengua materna una lengua vernácula o no. Esta figura evidencia tres resultados. El primero es que la brecha entre indígena y no indígena en cuanto al acceso a la educación primaria se ha reducido continuamente durante estas cuatro décadas hasta ser casi nula para la última generación (diferencia de 7 puntos). El segundo, más pesimista, es que la diferencia entre indígena y no indígena ha seguido creciendo en términos de acceso a la educación secundaria y superior, con excepción de la última generación. Por último, la discriminación contra la población indígena es más fuerte en el acceso a la educación secundaria (la distancia entre indígenas y no indígenas para la última generación es de casi 30 puntos).

(11) La educación bilingüe está todavía muy poco desarrollada en el Perú.

(12) Además, el análisis sólo se refiere a los jefes de familia y a sus cónyuges.

(13)Ya que no depende de la percepción que la persona tiene de sí misma. 


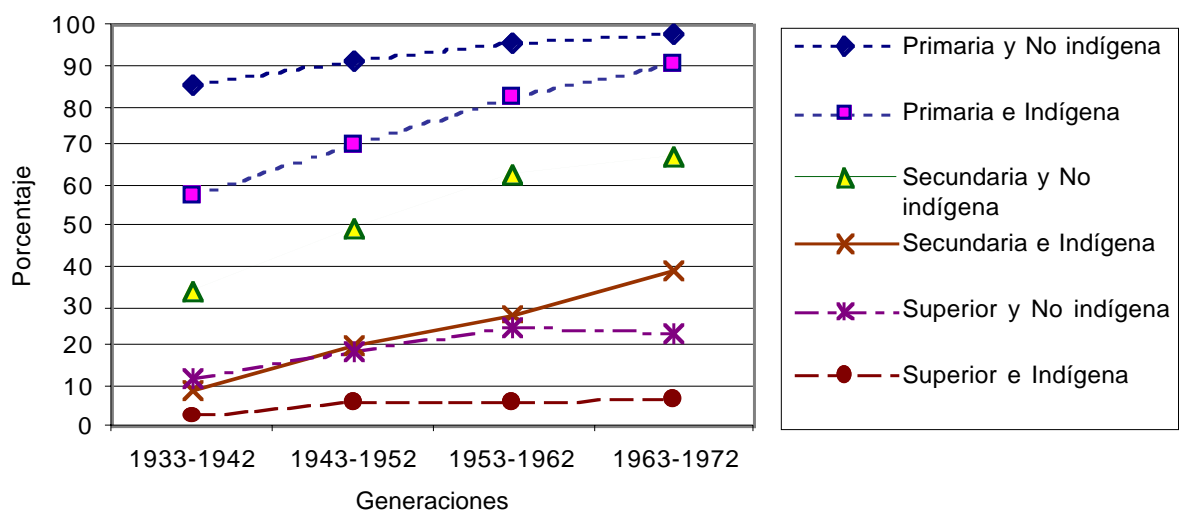

Fuente: encuesta ENAHO 2001.

Fig. 5 - Evolución del porcentaje de los que alcanzaron la primaria, la secundaria y la educación superior, según el criterio étnico en su definición limitada.

Se busca ahora controlar el efecto de la presencia más o menos importante de infraestructuras escolares en el momento en que la persona se educó. Si, por ejemplo, las personas indígenas que viven en la Sierra, donde hay mucho menos infraestructuras que en la Costa, migran a Lima para trabajar, se observará que los indígenas que se encuentran en Lima se educan menos que los no indígenas de Lima, pero esto puede explicarse por el hecho de que consiguieron su escolaridad en un lugar donde la oferta de escolaridad era escasa. Es posible controlar este efecto para las personas nacidas en Lima (14). Si se observa que, entre las personas nacidas en Lima — que aprovecharon infraestructuras escolares desarrolladas-, el acceso a la educación de los indígenas es más reducido que el de los no indígenas, se puede hablar de discriminación. Para distinguir a las personas según su lugar de nacimiento, se tuvo que adoptar la definición más amplia de indígena - uno de los padres o abuelos tiene por lengua materna una lengua vernácula - ya que no hay suficiente personas indígenas en Lima que respondan a las características de la definición más limitada para poder hacer un estudio robusto.

La figura 6 presenta los porcentajes de éxito de las personas nacidas en Lima según sean indígenas —en sentido amplio — o no. Muestra que el crecimiento de la cobertura de la educación primaria fue más rápido para la población indígena que para la población no indígena, reduciéndose la distancia entre estas dos poblaciones. Para la

(14) Para poder ampliar la prueba al conjunto del territorio, y no solamente Lima, sería necesario saber para cada persona el grado de desarrollo de las infraestructuras de su lugar de nacimiento en el momento de su educación y asegurarse de que las personas no migraron en el transcurso. A continuación, se puede comparar sólo el éxito escolar de la población indígena con el de la población no indígena para personas que tienen la misma oferta de educación. Desgraciadamente, no disponemos de tal información. 
primera generación, esta distancia era de más de 15 puntos. Sólo es de 5 puntos para la última generación. Así pues, los indígenas de la última generación nacidos en Lima acceden a la casi universalidad de la educación primaria, universalidad que beneficia a los no indígenas desde la generación nacida entre 1933-1942.

Las probabilidades de los indígenas — según la definición amplia— de acceder a la educación secundaria se han vuelto las mismas que las de los no indígenas para las generaciones nacidas entre 1943 y 1962, pero la distancia entre indígena y no indígena de nuevo aumentó para la última generación.

Además, las diferencias siguen siendo muy marcadas cuando se trata del acceso a la educación superior y tienden más bien a incrementarse con el tiempo, en Lima. Entre los de la última generación que nacieron en Lima, un 45\% de los no indígenas sobrepasó la educación secundaria mientras que esta proporción es sólo del $24 \%$ para los indígenas en el sentido más amplio. En Lima, la discriminación parece, pues, muy fuerte en el acceso a la educación superior y los migrantes de la segunda generación o más tienen mucho menos probabilidades de tener educación superior que un limeño no indígena. Para concluir con más robustez, sería necesario profundizar en el análisis, midiendo otras características intrínsecas como el nivel de vida de los padres. Pero éste no es el objeto de este artículo.

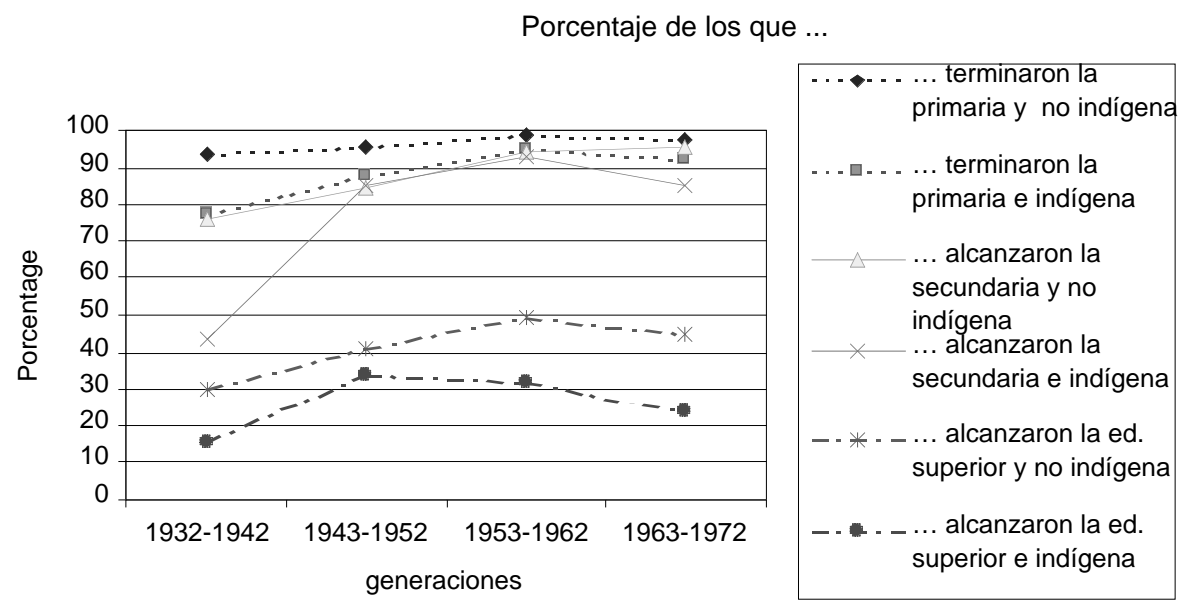

Fuente: encuesta ENAHO 2001.

Lectura: entre las personas nacidas entre 1932 y 1942 y definidas como indígenas, un 78\% terminaron la primaria y un 30\% alcanzaron la educación superior.

Fig. 6 - Porcentaje de los que terminan la primaria, que alcanzan la educación secundaria o superior según el criterio étnico en su definición amplia. 


\section{5. Conclusión acerca de la evolución de la cobertura del sistema educativo}

Las características de la expansión del sistema educativo peruano, evidenciadas por el análisis anterior, son las siguientes:

- A lo largo del siglo XX, el Perú conoció una expansión muy acentuada de su sistema educativo.

- La enseñanza primaria se volvió casi universal, excepto en el medio rural. Un estudio realizado en trece países industrializados (15) y dirigido por Blossfeld \& Shavit (1993) pone en evidencia que, en todos estos países, la educación primaria se ha vuelto universal y que la transición que diferencia a las personas parte de la decisión de proseguir o no estudios secundarios. En cambio, en el medio rural en Perú, y especialmente para las mujeres, la decisión discriminatoria consiste en permitir o no a los niños terminar su educación primaria.

- El desarrollo de la educación superior fue muy rápido y siguió de cerca la evolución de la demanda de trabajo calificado, con, sin embargo, un exceso de personas calificadas en las ciudades.

- Ocurrió una reducción sustancial de las diferencias entre los sexos. Son las mujeres las que han aprovechado más la expansión del sistema, excepto en el sector rural. Dicha disminución de las desigualdades entre los sexos es una tendencia que se observa también en los países desarrollados (Blossfeld \& Shavit, 1993).

- Las desigualdades entre los indígenas, definidos por el hecho de tener como lengua materna una lengua vernácula, y los no indígenas se redujeron en lo que se refiere al acceso a la escuela primaria, pero se han intensificado en el acceso a la educación superior.

\section{LA EVOLUCIÓN DEL PAPEL DEL ORIGEN SOCIAL EN EL ÉXITO ESCOLAR}

El análisis anterior de la expansión del sistema educativo puso en evidencia que las desigualdades en los niveles escolares disminuyeron, aunque no se redujeron para todos con la misma intensidad. Una pregunta sigue quedando pendiente: ¿la masificación de la escolaridad fue acompañada de una reducción de las diferencias de niveles de educación entre las personas de diferente origen social? o ¿no fue más que una traslación de los niveles escolares hacia arriba, sin que se haya modificado la desigualdad de oportunidades?

Se entiende aquí por "igualdad de oportunidades" el concepto definido por Roemer (1998), lo que se basa en el principio de "compensación de las desventajas" (16). Según este principio, existe igualdad de oportunidad cuando se compensa a las personas que sufren de una desventaja cualquiera vinculada a su origen social

(15) Los Estados Unidos, la ex RFA, Holanda, Suecia, Inglaterra, Escocia, Italia, Suiza, Taiwán, Japón, Polonia, Hungría, Checoslovaquia e Israel.

(16) La igualdad de oportunidad puede verse de dos maneras diferentes. La primera es la que Roemer denominó el principio de no discriminación, existe igualdad de oportunidad si no se discrimina 
—dándoles más educación, por ejemplo_ con el fin de nivelar las diferencias entre los individuos. Las diferencias que deben nivelarse son las que son independientes de la voluntad del individuo o, dicho con palabras de Roemer, independientes del "esfuerzo" que proporciona, pero que resultan de sus "circunstancias", es decir, de factores sobre los cuales no tiene ninguna influencia, tal como sus genes, la educación de sus padres, las infraestructuras cercanas... En el caso de la educación, por ejemplo, algunos niños serán más aptos que otros a sacar provecho de la educación que se les proporciona y esta aptitud depende de sus circunstancias. Pero niños con aptitudes iguales pueden hacer esfuerzos diferentes, según su voluntad. Instaurar la igualdad de oportunidades consiste entonces en compensar a las personas en sus diferencias de circunstancias y no en las vinculadas al esfuerzo realizado.

El objetivo de esta parte consiste en determinar en qué medida se ha modificado, en el Perú en el siglo XX, el efecto sobre el éxito escolar de una persona de las circunstancias vinculadas a su origen social o, más precisamente, a su origen cultural. Para ello, es necesario comenzar por definir una medida del vínculo entre el origen cultural y el éxito escolar, es decir, una medida de la movilidad escolar. Es el objetivo de la primera subsección de esta parte. Luego, en la segunda subsección, se describirá, con ayuda de la medida escogida, cómo se ha modificado el vínculo global entre el origen cultural y el éxito escolar durante el siglo XX. Finalmente, en la tercera subsección, se descompondrá este vínculo con el fin de extraer el componente que traduce el grado de igualdad de oportunidades escolares.

\section{1. La elección de una medida de la movilidad escolar}

No existe en la literatura ningún consenso sobre la elección de una medida de la movilidad escolar, ya que las medidas seleccionadas dependen del aspecto de la movilidad que el autor busca destacar y del tipo de datos de que dispone. Por esta razón, la lista de las medidas utilizadas es larga. Sin embargo, se pueden distinguir tres grandes tipos de medida. El primer tipo, quizás el más utilizado (Bourguignon et al., 2001; Behrman et al., 2001; Anderson, 2001a), está constituido por las medidas construidas a partir del coeficiente $\beta$ de una cadena de Markov de primer orden. $\beta$ puede definirse como sigue:

$$
E_{i, t}=\alpha+E_{i, t-1} \beta+u_{i, t} \quad, \beta \geq 0
$$

en donde $E_{i, t}$ es el nivel de estudio de la generación $t$ (los hijos) de la familia $i$ y $E_{i, t-1}$

el nivel de estudio de la generación $t$-1 (los padres) de la familia $i, \alpha$ una constante. Cuanto más el coeficiente $\beta$ es elevado, más el nivel de educación de los padres determina el de los hijos, es decir, más el grado de movilidad escolar es débil. Si los niveles de educación se miden de la misma forma para los padres que para los hijos, un valor de $\beta$ igual a 1 significa una perfecta reproducción de desigualdades en los niveles de educación alcanzados. Si $\beta<1$, las diferencias en los niveles de educación disminuirán

de ninguna manera (según criterios de raza, sexo, ...) para acceder a una posición dada. Sólo las características que forman parte de la posición dada deben tenerse en cuenta. La segunda manera de concebir la igualdad de oportunidad es aquélla que, a ejemplo de Roemer, se retiene aquí, la de “compensación de las desventajas". Esta definición es mucho más amplia que la primera. 
entre las generaciones hasta cancelarse, todos los niveles convergiendo hacia el promedio. Entre las medidas construidas a partir de $\beta$, se encuentra el coeficiente de correlación de Pearson, $\rho$, que muy a menudo se emplea en la literatura (Binder \& Woodruff, 2002). Este tipo de medida tiene dos inconvenientes: sólo tiene sentido con variables continuas y no permite relación no linear. Por ejemplo, estas medidas evalúan siempre de la misma manera el aumento de dos años entre el nivel escolar del padre y el de los hijos, mientras que se podría pensar que pasar, de una generación a otra, de un nivel de enseñanza primaria incompleta a un nivel de enseñanza primaria completa es un salto mayor que pasar de cinco años de universidad a siete años de universidad. Por estas razones, no se escogió este tipo de medida aquí. Los dos demás tipos de medidas permiten precisamente relaciones no lineares y asimétricas y pueden construirse a partir de variables categoriales. El segundo tipo de medidas son medidas de asociación global construidas a partir de la estadística de $\chi^{2}$. El $\chi^{2}$ mide la distancia entre la frecuencia de las células de la tabla de contingencia conformada por las variables $E_{i, t-1}$ y $E_{i, t}$ y la frecuencia teórica en caso de independencia total de las dos variables (17). El problema del $\chi^{2}$ es que es sensible al tamaño de la muestra. Esta es la razón por la que se utiliza generalmente el V de Cramer (18) (Thélot \& Vallet, 2000) que permite comparar tablas que tienen un número de observaciones diferente.

El tercer tipo de medidas son los índices axiomáticos elaborados a partir de las matrices de transición (19) (Checchi et al., 1999). Un índice axiomático de movilidad es un escalar que sintetiza la información contenida en las matrices de transición y que se define de modo que compruebe algunos axiomas. Los axiomas y los distintos índices axiomáticos que se utilizarán aquí se presentan en el Anexo 1.

\section{2. Evolución del vínculo global entre el origen cultural y el éxito escolar}

Las distintas medidas de movilidad escolar seleccionadas — del segundo y del tercer tipo- no resultan forzosamente en la misma clasificación de las matrices de transiciones de cada generación, puesto que cada una hace resaltar un aspecto diferente de la movilidad. No existe, sin embargo, ninguna razón para favorecer a una. Por ello, se tendrán todas en cuenta y sólo se comentarán los resultados robustos a las elecciones de la medida. Todas las medidas concuerdan en los puntos siguientes (Fig. 7).

En las ciudades, el vínculo global entre el origen social y el éxito escolar se ha

(17) La frecuencia teórica de la célula formada por la línea $i$ y la columna $j$ en caso de independencia es igual al producto del margen de la línea $i$ con la de la columna $j$, dividido por el número total de observaciones.

(18) El V de Cramer se expresa así: $V=\chi^{2} /(n(k-1))$ en donde $n$ es el número de observaciones y $k$ la más pequeña dimensión de la tabla de contingencia.

(19) Una matriz de transición es una matriz $\left(n_{i}, n_{j}\right)$ en donde $n_{i}$ y $n_{j}$ son el número de estados iniciales y el número de estados finales posibles, respectivamente. Está constituida por los elementos $p_{i j}$, que son la probabilidad de alcanzar el estado $j$ (columna) cuando se viene del estado $i$ (línea). Para una matriz intergeneracional entre padre e hijos, $i$ será la situación del padre, $j$ la del hijo y $p_{i j}$ la probabilidad para un individuo de alcanzar el estado $j$, sabiendo que su padre viene del estado $i$. La suma de los elementos de una línea $i$ de la matriz de transición es igual a $1\left(\sum_{j} p_{i j}=p_{i .}=1\right)$. 

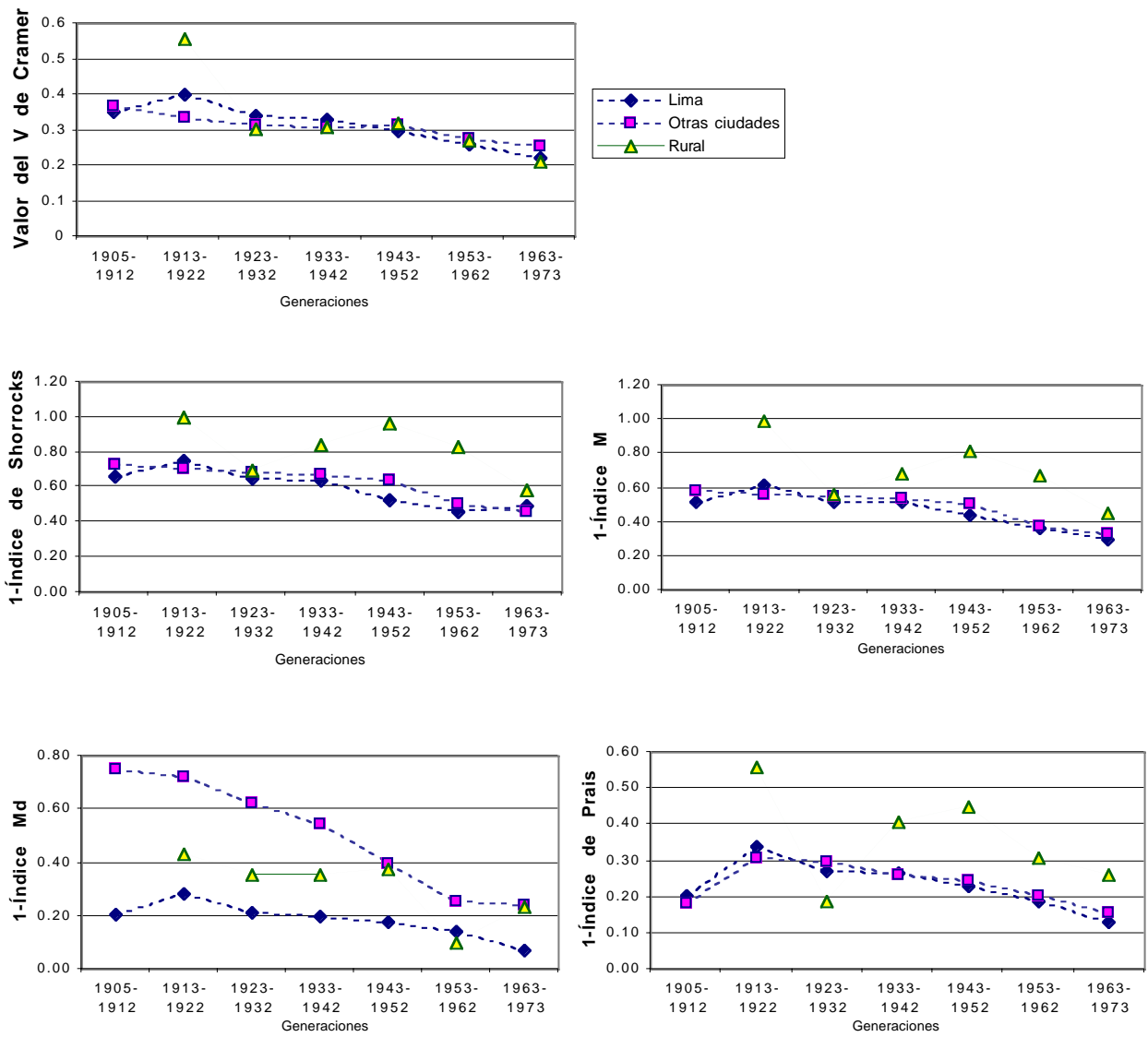

Fuentes: encuestas 1974, 1985-1986, 1990, 1996, 2001.

Fig. 7 - Evolución de la movilidad escolar según distintos índices.

venido debilitando de manera continua a partir de los años de educación de la generación nacida en 1913-1922. Anteriormente, la movilidad tendía a reducirse en Lima. Nada se puede afirmar sobre lo que sucedía en otras ciudades durante este periodo por no contar con medidas coherentes.

En el medio rural se observan tres periodos diferentes. En el primer periodo, entre la generación nacida entre 1913 y 1922 y aquélla nacida entre 1923 y 1932, el vínculo se distendió mucho, pero se volvió a afianzar, en el segundo periodo, de manera monótona para las generaciones nacidas entre 1933 y 1952. Finalmente, se aflojó nuevamente entre la generación nacida entre 1943 y 1952 y la nacida entre 1953 y 1962 -y quizás entre la última y la penúltima generación, pero el índice Md muestra el contrario-. 
Las medidas de la movilidad no nos permiten decir si el vínculo es más fuerte en Lima, en las otras ciudades o en el sector rural, pues cada una genera una clasificación diferente.

Cuando se estudia este vínculo diferenciando los sexos, se percibe que este vínculo es el mismo para las niñas y los niños y que se transforma de la misma forma con el tiempo, cualquiera sea la medida de la movilidad elegida.

La evolución del vínculo global entre el origen cultural y social y el éxito escolar parece explicarse sólo parcialmente por la prolongación del tiempo de estudios. En efecto, el debilitamiento del vínculo corresponde, en general, a una expansión del sistema escolar - tanto del tiempo promedio de estudios como del aumento del alumnado en la educación primaria y superior - aún cuando las intensidades de variaciones no necesariamente corresponden. Sin embargo, se observan algunas excepciones: en Lima, la primera generación se ve beneficiada por un importante desarrollo del sistema escolar - la población escolar que termina la primaria aumenta de un $20 \%$, la que termina los estudios superiores de un $33 \%, \ldots-$. Sin embargo el vínculo global entre el origen social y el éxito escolar se consolida —aumentó del 13\% al 68\% según los índices-. Igualmente, en el medio rural, el sistema escolar y el vínculo global evolucionan de la misma manera para las generaciones nacidas entre 1923 y 1952: un acceso más generalizado a la educación se acompaña de la consolidación del lazo intergeneracional. Finalmente, la flexibilización del vínculo global es muy marcada en el medio rural para la generación nacida entre 1913 y 1922 aunque, en este mismo periodo, el sistema escolar se desarrolló poco. Así, la expansión del sistema escolar no explica sino parcialmente la distensión del vínculo entre el origen y el éxito escolar. El objetivo de la siguiente parte es determinar cuál es el papel de la prolongación de los estudios con respecto a la evolución del grado de igualdad de oportunidades en la evolución del vínculo global.

\section{3. Evolución de la desigualdad de oportunidades en el éxito escolar}

\section{3. 1. La problemática}

La evolución de la movilidad escolar intergeneracional es el resultado de dos fenómenos bien distintos: la prolongación general de los estudios y la evolución de las probabilidades relativas de éxito escolar, que se nombrará también el vínculo "puro" entre el origen cultural y el éxito escolar. Este vínculo "puro" traduce el grado de igualdad de oportunidades que puede observarse en la sociedad. Si existe una perfecta igualdad de oportunidades o, en otras palabras, si el vínculo "puro" entre el origen cultural y el éxito escolar es nulo, las diferencias entre los niveles escolares sólo pueden explicarse por las opciones elegidas — particularmente, la de hacer o no un esfuerzoy, en ningún caso, por características ajenas a su voluntad, como su entorno familiar.

Se puede imaginar, por ejemplo, que el vínculo global entre el origen cultural y el éxito escolar se distiende sólo como consecuencia del aumento general de la escolaridad. El aumento general de la escolaridad acrecienta las diferencias entre la educación de los hijos y la de sus padres, de donde resulta un vínculo global más débil. Sin embargo, la probabilidad de que una persona nacida en un entorno de poca 
educación alcance el mismo nivel de educación que otra nacida en un entorno muy instruido seguirá siendo la misma. Si el vínculo "puro" sigue siendo el mismo, la evolución de la distribución de los niveles escolares no es más que una traslación hacia arriba.

Para ilustrar esto, podemos suponer dos sociedades, una sociedad A y una sociedad B. Ambas tienen, en $t$, la misma distribución de niveles escolares en función del origen, representada por la matriz de transición $x$. En las filas de esta matriz están representados los niveles escolares de los padres y, en las columnas, la de los hijos. Tres niveles escolares son posibles — sin educación o primaria, secundaria, superior- - La matriz $x$ traduce una situación de inmovilidad total y completa desigualdad de oportunidades: los hijos tienen una probabilidad nula de tener un nivel escolar diferente del de su padre. En las dos sociedades, el vínculo global entre la educación del padre y la de los hijos se relaja. En $t+1$, la sociedad A se caracteriza por la matriz de transición $y$, la sociedad B por $z$ :

$$
\begin{array}{ll}
\text { Sociedad A : } & x=\left(\begin{array}{lll}
1 & 0 & 0 \\
0 & 1 & 0 \\
0 & 0 & 1
\end{array}\right) \rightarrow y=\left(\begin{array}{ccc}
0,5 & 0,5 & 0 \\
0 & 0,5 & 0,5 \\
0 & 0 & 1
\end{array}\right) \\
\text { Sociedad B : } & x=\left(\begin{array}{lll}
1 & 0 & 0 \\
0 & 1 & 0 \\
0 & 0 & 1
\end{array}\right) \rightarrow z=\left(\begin{array}{lll}
0 & 1 & 0 \\
0 & 1 & 0 \\
0 & 1 & 0
\end{array}\right)
\end{array}
$$

En la sociedad A, el debilitamiento del vínculo se explica casi enteramente por la prolongación de los estudios. En $t$, una persona tiene una probabilidad nula de alcanzar un nivel escolar diferente del de su padre mientras que en $t+1$ tiene un $50 \%$ de posibilidades de obtener el mismo nivel que su padre y un 50\% de superarlo, cuando es factible. El tiempo de estudio se ha prolongado en general, ya que uno de cada dos hijos tendrá un nivel superior al de su padre (20)y los otros el mismo nivel, pero en $t+1$ el nivel escolar alcanzado sigue dependiendo del origen social de la persona. Por ejemplo, las personas cuyo padre tiene un nivel superior no tienen ninguna probabilidad de interrumpir sus estudios al nivel primario mientras que las cuyo padre sólo tiene un nivel primario no tienen ninguna probabilidad de alcanzar la educación superior. Por el contrario, en la sociedad B, el vínculo se distendió únicamente por una dependencia menor entre el nivel escolar de los hijos y el de sus padres. En efecto, el tiempo promedio de estudio se mantuvo entre $t$ y $t+1$ (21), puesto que las personas superan el nivel de su padre si este último es bajo y tienen un nivel inferior si es elevado. Además, todas las personas tienen las mismas probabilidades de alcanzar un nivel escolar dado, cualquiera

(20) Cuando es factible. categorías.

(21) A condición de que la población se distribuya de manera igual entre las distintas 
sea su origen: tienen un $100 \%$ de probabilidades de alcanzar el nivel secundario. Así pues, la distensión del vínculo en la sociedad B se explica por un grado más importante de igualdad de oportunidades.

La pregunta a la que se pretende responder ahora es ¿cuál es el porcentaje de distensión del vínculo global entre el origen social y el éxito escolar que se explica por la prolongación de los estudios y la que se explica por la flexibilización del vínculo "puro" entre el nivel escolar del hijo y el del padre? Los modelos log-lineales permiten contestar esta cuestión.

\section{3. 2. Los modelos}

El primer modelo, el modelo (1), supone que el único factor que ha evolucionado en el tiempo es la distribución de los niveles escolares, tanto el de los padres como el de los hijos. Pues, este modelo permite verificar la hipótesis según la cual el vínculo entre el éxito escolar y el origen cultural se ha mantenido estable en el tiempo. Así, según este modelo, las matrices de transición intergeneracionales de los niveles de educación se transformaron con el tiempo bajo el efecto de un factor único, la prolongación general del tiempo de estudio, manteniéndose sin variación la relación entre entorno de origen y éxito escolar.

El modelo (2) es igual al primero, excepto que permite que el vínculo entre el origen cultural y el éxito escolar varíe de una generación a la otra. El modelo (2) incluye entonces el modelo (1) y una triple interacción entre la educación de la persona, su origen social y su generación. Permite identificar si las desigualdades sociales en la escuela se modificaron con el tiempo. La variación del vínculo que supone es uniforme, es decir que, en el paso de una generación a otra, todas las relaciones de las probabilidades relativas — "odds ratio"- varían en el mismo sentido y con la misma intensidad, medida por un parámetro $\beta$ (véase Anexo 2). Si el modelo (2) se ajusta mejor a los datos empíricos que el modelo (1), esto significa que la evolución de los niveles escolares tiene explicación no sólo por la masificación de la educación, sino también por una democratización de la misma.

Se presentan estos dos modelos con más detalle en el Anexo 2. Varios indicadores permitirán evaluar los modelos. Se encuentran en el cuadro 7 para Lima, el cuadro 8 para las otras ciudades y el cuadro 9 para las zonas rurales. El mejor modelo será el que calce mejor con los datos observados y que contenga, al mismo tiempo, la menor cantidad de parámetros. La facultad de encaje del modelo es considerada por la estadística de verosimilitud ( $G^{2}$ ) y el número de parámetros que contenga por su grado de libertad $(d d l)$. El índice BIC permite tener en cuenta simultáneamente estos dos criterios. Más reducido es el BIC, mejor es el modelo. 


\section{Cuadro 7 - Indicadores de ajuste de los modelos para Lima.}

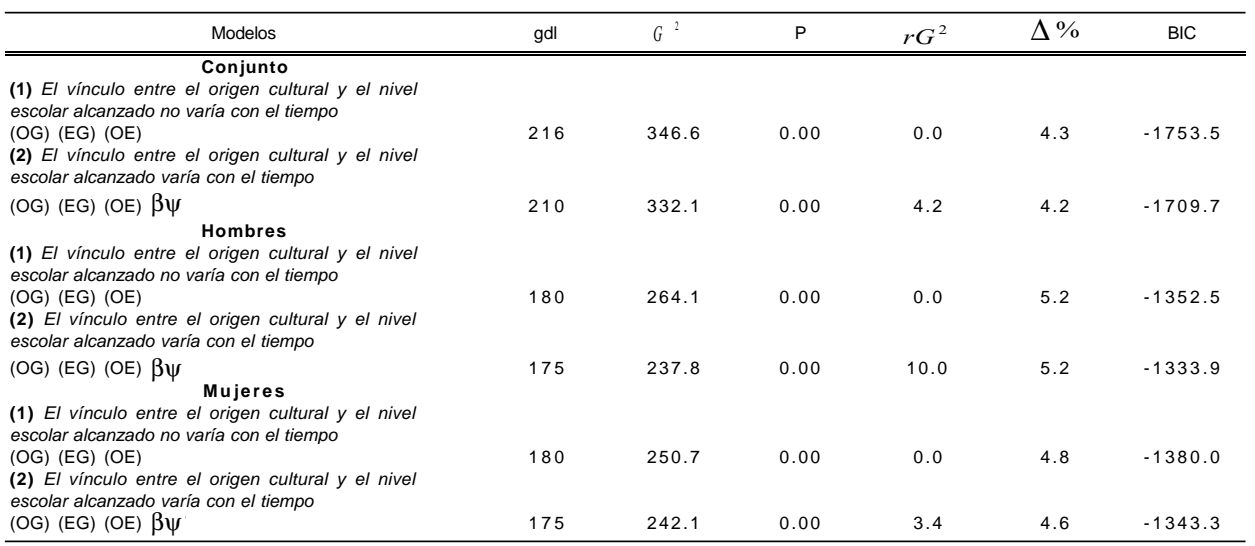

Fuentes: encuestas 1974, 1985-1986, 1990, 1996, 2001.

Campo: Lima, los 23 a 69 años. Para el conjunto, el análisis incluye las siete generaciones. Para los hombres y las mujeres considerados separadamente, no se tiene en cuenta la primera generación (muy poca representatividad).

Lectura: la adecuación del modelo a los datos empíricos se mide por la estadística de verosimilitud $G^{2}$. $G^{2}$ sigue asintoticamente una ley de $\chi^{2}$ a $d d l$ grados de libertad. Si $\mathrm{p}<0,05$, la hipótesis según la cual los datos estimados por el modelo no son significativamente diferentes de los datos empíricos se desestima al umbral del 5\%. $r G^{2}$ da el porcentaje de la distancia entre el modelo (1) y los datos empíricos que explica el modelo en cuestión. Por ejemplo, para el modelo (2), $\quad r G_{(2)}^{2}=100-100 G_{(2)}^{2} / G^{2}$. $\Delta \%$ es la proporción del número total de personas que debería cambiarse de casilla para que la tabla de datos estimados sea igual a la tabla de datos observados. El número de personas que debería cambiarse de casilla, $\Delta$, es igual a la semisuma de los valores absolutos de las diferencias, en cada casilla, entre la frecuencia observada y la frecuencia estimada. Ninguno de los modelos evalúa suficientemente bien los datos para que la diferencia entre los datos estimados y los datos empíricos sea significativamente nula. Es la razón por la que se introduce el criterio del BIC que tiene en cuenta los tres parámetros $G^{2}, d d l$ y $N$, el número total de personas de la tabla. Este criterio permite afirmar que un modelo debe preferirse al otro si su BIC es más reducido —al menos por 6 puntos-. El indicador del BIC se define así: $B I C=G^{2}-d d l \ln (N)$. Según el criterio del BIC, se debe escoger el modelo (1). 


\section{Cuadro 8 - Indicadores de ajuste de los modelos para las zonas urbanas fuera de Lima.}

\begin{tabular}{|c|c|c|c|c|c|c|}
\hline Modelos & gdl & $G^{2}$ & $\mathrm{P}$ & $r G^{2}$ & $\Delta \%$ & $\mathrm{BIC}$ \\
\hline $\begin{array}{l}\text { Conjunto } \\
\text { (1) El vínculo entre el origen cultural y el nivel } \\
\text { escolar alcanzado no varía con el tiempo } \\
\text { (OG) (EG) (OE) } \\
\text { (2) El vínculo entre el origen cultural y el nivel } \\
\text { escolar alcanzado varía con el tiempo }\end{array}$ & 216 & 543.1 & 0.00 & 0.0 & 3.4 & -1723.5 \\
\hline $\begin{array}{l}\text { (OG) (EG) (OE) } \beta \psi \\
\text { (1) El vínculo entre el origen cultural y el nivel }\end{array}$ & 210 & 509.3 & 0.00 & 6.2 & 3.3 & -1694.3 \\
\hline $\begin{array}{l}\text { (OG) (EG) (OE) } \\
\text { (2) El vínculo entre el origen cultural y el nivel } \\
\text { escolar alcanzado varía con el tiempo }\end{array}$ & 180 & 373.9 & 0.00 & 0.0 & 3.8 & -1380.3 \\
\hline $\begin{array}{l}\text { (OG) (EG) (OE) } \beta \psi \text { Mujeres } \\
\text { (1) El vínculo entre el origen cultural y el nivel } \\
\text { escolar alcanzado no varía con el tiempo }\end{array}$ & 175 & 348.7 & 0.00 & 6.7 & 3.7 & -1356.8 \\
\hline $\begin{array}{l}\text { (OG) (EG) (OE) } \\
\text { (2) El vínculo entre el origen cultural y el nivel } \\
\text { escolar alcanzado varía con el tiempo }\end{array}$ & 180 & 396.2 & 0.00 & 0.0 & 4.3 & -1375.2 \\
\hline$(\mathrm{OG})(\mathrm{EG})(\mathrm{OE}) \quad \beta \psi$ & 175 & 378.2 & 0.00 & 4.5 & 4.2 & -1344.0 \\
\hline
\end{tabular}

Fuentes: encuestas 1974, 1985-1986, 1996, 2001.

Campo: Zonas urbanas fuera de Lima, los 23 a 69 años. Para el conjunto, el análisis incluye las siete generaciones. Para los hombres y las mujeres considerados separadamente, no se tiene en cuenta la primera generación (muy poca representatividad).

Cuadro 9 - Indicadores de ajuste de los modelos para las zonas rurales.

\begin{tabular}{|c|c|c|c|c|c|c|}
\hline Modelos & gdl & $G^{2}$ & $P$ & $r G^{2}$ & $\Delta \%$ & $\mathrm{BIC}$ \\
\hline $\begin{array}{l}\text { (1) El vínculo entre el origen cultural y el nivel } \\
\text { escolar alcanzado no varía con el tiempo } \\
\text { (OG) (EG) (OE) } \\
\text { (2) El vínculo entre el origen cultural y el nivel } \\
\text { escolar alcanzado varía con el tiempo }\end{array}$ & 180 & 263.5 & 0.00 & 0.0 & 3.6 & -1440.2 \\
\hline $\begin{array}{l}\text { (OG) (EG) (OE) } \beta \psi \text { Hombres } \\
\text { (1) El vínculo entre el origen cultural y el nivel } \\
\text { escolar alcanzado no varía con el tiempo }\end{array}$ & 175 & 217.3 & 0.00 & 17.5 & 3 & -1439.1 \\
\hline $\begin{array}{l}\text { (OG) }(\mathrm{EG})(\mathrm{OE}) \\
\text { (2) El vínculo entre el origen cultural y el nivel } \\
\text { escolar alcanzado varía con el tiempo }\end{array}$ & 180 & 252.8 & 0.00 & 0.0 & 4.9 & -1325.2 \\
\hline $\begin{array}{l}\text { (OG) (EG) (OE) } \beta \psi \text { Mujeres } \\
\text { (1) El vínculo entre el origen cultural y el nivel } \\
\text { escolar alcanzado no varía con el tiempo }\end{array}$ & 175 & 222.9 & 0.00 & 11.8 & 4.4 & -1311.3 \\
\hline $\begin{array}{l}\text { (OG) (EG) (OE) } \\
\text { (2) El vínculo entre el origen cultural y el nivel } \\
\text { escolar alcanzado varía con el tiempo }\end{array}$ & 180 & 185.8 & 0.00 & 0.0 & 4.3 & -1393.8 \\
\hline$(\mathrm{OG})(\mathrm{EG})(\mathrm{OE}) \beta \psi$ & 175 & 130.6 & 0.00 & 29.7 & 2.7 & -1405.1 \\
\hline
\end{tabular}

Fuentes: encuestas 1985-1986, 2001.

Campo: Zonas rurales, los 23 a 69 años. El análisis se refiere a seis generaciones, la de mayor edad no ha sido cubierta por las encuestas.

\section{3. 3. Los resultados}

Los modelos (1) y (2) reproducen bien los datos, puesto que sólo alrededor del $4 \%$ del número total de personas de la tabla de datos estimados debe desplazarse para 
llegar a la tabla de datos reales en casi todas las tablas (véase $\Delta \%$ ). En el medio urbano - Lima y las otras ciudades_-, tanto para los hombres como para las mujeres, el modelo (2) reproduce mejor los datos, pero el beneficio en verosimilitud no justifica la pérdida de los grados de libertad, dado que el BIC del modelo (1) es más reducido que el del modelo (2). Se retiene, pues, el modelo(1) que supone que las relaciones de probabilidades relativas son invariantes en el tiempo. Lo mismo ocurre con la categoría de los hombres en el medio rural: el modelo (1) es preferible al modelo (2). Por el contrario, para las mujeres en zonas rurales, el modelo (2) que tiene en cuenta una evolución uniforme de las relaciones de probabilidades es preferible al modelo (1). Los parámetros $\beta_{k}$ estimados por el modelo (2) muestran un fuerte aflojamiento del vínculo "puro" entre el origen cultural y el éxito escolar.

Así, para los hombres en general y para las mujeres en medio urbano, alcanzar un determinado nivel de educación depende tanto hoy como a principios del siglo XX de la educación de los padres aun cuando la educación se ha vuelto accesible a un mayor número de personas y que, por lo tanto, la desigualdad en los niveles de educación ha disminuido. Dicho de otro modo, si bien se observa que un $70 \%$ de la generación de mayores de edad cuyo padre no asistió a la escuela no lo hizo tampoco, para las generaciones más jóvenes, esta proporción es sólo del 11\% (22).Esto se explica por el aumento considerable de la cobertura escolar que el Perú ha alcanzado a lo largo del siglo XX, pero la probabilidad de una persona cuyo padre tuvo un bajo nivel escolar de alcanzar el nivel escolar de una persona cuyo padre sí tuvo un alto nivel escolar sigue siendo la misma de una generación a otra. Las desigualdades en los niveles de educación han disminuido mucho durante el siglo, pero la desigualdad de oportunidades sigue siendo la misma, cabe decir que no existe una verdadera democratización de la educación, con la notable excepción de las mujeres en el medio rural. Para estas últimas, por el contrario, existe un fuerte aumento de la igualdad de oportunidades.

El incremento constante de la movilidad en el transcurso de las generaciones que expresaban los índices de movilidad no tradujo realmente sino la distensión del vínculo entre la educación del padre y la educación del hijo por un crecimiento más rápido de los niveles de educación de los hijos con respecto a los de los padres.

Este resultado que se pone en evidencia para el Perú, es decir, que el grado de oportunidad escolar ha sido el mismo a lo largo del siglo, concuerda con el que se encontró en líneas generales para los países desarrollados (Blossfeld \& Shavit, 1993).

\section{4. ¿QUÉ POLÍTICAS APLICAR PARA AUMENTAR LA IGUALDAD DE OPORTUNIDADES ESCOLARES?}

Para responder a esta pregunta esencial, es necesario analizar en primer lugar cuáles son los factores de bloqueo que hacen que la expansión del sistema educativo no se acompañe de un aumento de la igualdad de oportunidades. Pueden evidenciarse dos tipos de factores. Por una parte, los factores vinculados a la oferta de educación escolar y, por otra parte, los vinculados a la demanda de educación escolar. Si la oferta de

(22) Es lo que se observa en Lima. 
educación —infraestructuras escolares, número de profesores...- no es la misma para todos, entonces se crea un primer tipo de bloqueo a la igualdad de oportunidades escolares. Por ejemplo, cuando el Estado decide invertir menos en el medio rural porque su inversión es menos rentable — es necesario construir una escuela en cada comunidad que agrupa a pocos alumnos, los profesores se niegan a enseñar en zonas aisladas sin compensación salarial...-, se discrimina a los niños que nacen en zonas rurales con relación a los que nacen en la ciudad, a menos que los primeros decidan migrar para educarse. Además de analizar la evolución del gasto público en educación desde los años 30, Saavedra \& Melzi (1998) demuestran que en el Perú, en 1994, la asignación de los recursos educativos del Estado por alumno se correlaciona negativamente con el grado de pobreza del departamento. Más pobre es el departamento (23), más el gasto por alumno en el departamento es reducido. En el caso de bloqueo vinculado a la oferta de educación, las políticas públicas deben mejorar la distribución del gasto público.

Los factores vinculados a la demanda de educación pueden ser de naturaleza muy diferente. Se distinguirán aquí tres grandes factores. El primero es aquél que analizan con todo detalle Becker \& Tomes (1986): en el contexto de mercados de crédito imperfectos — como es el caso en el Perú-, las familias pobres que no pueden endeudarse para financiar la educación de sus hijos, no tienen la capacidad económica de financiar la educación que desean para sus hijos - aunque la educación es gratuita, existen costos que no pueden asumir, tal como los materiales escolares, el uniforme, el transporte (24)y, sobre todo, el costo de oportunidad que representa enviar un hijo a la escuela cuando podría estar trabajando y contribuyendo al ingreso familiar-. El costo marginal de inversión en el capital humano de sus hijos es entonces más elevado que el de familias suficientemente acomodadas para endeudarse. Este argumento se acerca al de Boudon (1973) que diferencia los niveles escolares entre grupos sociales según un cálculo costo/beneficio. Alcanzar una posición elevada cuesta mucho más a los de medios desfavorecidos correlativamente a su nivel de vida que a los de medios favorecidos. Según estos dos modelos, la política que debe aplicarse es, pues, la de disminución de las desigualdades socioeconómicas entre las familias, proporcionando a las familias desfavorecidas la posibilidad de financiar estudios para sus hijos a través, por ejemplo, de un sistema de becas. Para Boudon (1973), por ejemplo:

"Si se admite que las desigualdades económicas son la dimensión más importante de la estratificación, se deduce del análisis que una reducción de las desigualdades económicas debe tener efectos importantes sobre la desigualdad de oportunidades frente a la educación." (p. 304).

Según estos autores, el origen socioeconómico es la razón que explica con mayor contundencia la desigualdad de oportunidades escolares.

(23) Los índices de pobreza por los que la correlación es significativa son el índice de pobreza de FONCODES, la tasa de analfabetismo femenino, la proporción de la población sin acceso a luz eléctrica, la tasa de ruralidad, el porcentaje de la población sufriendo de desnutrición crónica.

(24) Saavedra \& Suárez (2002) muestran que las familias se ven obligadas a aportar económicamente a la educación de sus hijos, aun cuando se supone que la educación primaria y secundaria es gratuita y accesible a todos, por el hecho de que el gasto público por alumno es muy reducido. En este sentido, las familias cubren la tercera parte del gasto público total en educación. 
Un segundo grupo de factores que frenan el aumento de la igualdad de oportunidades ante la enseñanza y que están vinculados a la demanda de educación es el que consiste en que los padres poco instruidos encuentran menor interés en educar a sus hijos que los padres con mayor nivel educativo. Una primera justificación la tiene la teoría del grupo de referencia (Merton, 1953). Según esta teoría, la ventaja que los padres poco instruidos ven en la inversión en la educación de sus hijos será, a partir de cierto umbral, menor que la de padres más instruidos. El argumento es que el objetivo de cada uno consiste en alcanzar o en superar ligeramente el nivel de educación de su grupo de referencia. Los padres con poca educación desearán para sus hijos un nivel ligeramente superior al suyo porque alcanzar niveles más altos les parece superfluo y hasta indeseable, ya que crearía una distancia social entre ellos y sus hijos. Al contrario, los padres con educación quieren que sus hijos alcancen un nivel de estudio al menos igual al suyo. Cercanos a esta teoría, Checchi et al. (1999) emiten la hipótesis según la cual la inversión en capital humano por parte de los padres dependerá de la creencia que tienen en las capacidades de sus hijos. Ahora bien, las capacidades no se conocen hasta después de pasar por la escuela. Si los resultados de una persona en la escuela son malos es que está dotada de bajas capacidades. Dado que las capacidades se transmiten con una determinada persistencia, los padres evaluarán las capacidades de sus hijos en relación con sus propias capacidades. Si fueron a la escuela y tuvieron éxitos, creerán que las capacidades de su hijo son elevadas; si fracasaron, que son pocas; y si no fueron a la escuela, los padres no tendrán idea de sus propias capacidades y considerarán que las de sus hijos son de un nivel promedio. A mayor fe en las capacidades del hijo, mayor es la inversión. En este sentido, el origen social tendrá una importancia determinante en el nivel escolar alcanzado por la persona. En estas dos argumentaciones, la dimensión cultural del origen influye de manera preponderante en la persistencia de la desigualdad de oportunidades. Si estas dos argumentaciones tienen fundamento, una política de ayuda financiera a las familias para la educación de sus hijos es útil pero poco influyente.

Por último, el tercer grupo de factores propuestos en la literatura es el que se inspira en la teoría del capital cultural de Bourdieu \& Passeron (1964; 1970). Según esta teoría, la educación perjudica a los niños que heredaron de un escaso capital cultural porque sus padres llegaron sólo hasta los niveles inferiores del sistema escolar, ya que no conocen los valores ni la lengua que se valorizan en la escuela. La escuela transmite los valores del grupo dominante que no son los suyos y esto los excluye rápidamente del sistema escolar. En este caso, las políticas de reducción de las desigualdades económicas no tienen absolutamente ninguna influencia en la desigualdad de oportunidades. Sólo una reforma del sistema escolar puede tener un efecto. En el caso del Perú, se puede pensar que esta teoría es vigente en el caso de las poblaciones indígenas. El sistema educativo heredado de los españoles transmite valores criollos que no son los de la población indígena y en una lengua que no es la suya. Una política podría ser entonces la de desarrollar la educación bilingüe en el Perú.

El objetivo de esta parte es aportar algunas luces sobre la eficacia de las distintas políticas públicas posibles. En primer lugar, preguntándose qué políticas podrían reducir los bloqueos procedentes de la oferta de educación. Para eso, se evaluarán cuáles fueron las consecuencias en términos de disminución de desigualdad de oportunidades de los esfuerzos hechos por el Gobierno durante el siglo XX en cuanto a educación. 
Seguidamente, se busca determinar cuál de las dimensiones de origen, es decir, de origen cultural o de origen socioeconómico, influye mayormente en la persistencia de la desigualdad de oportunidades escolares para evaluar la eficacia de una política de redistribución, con un sistema de becas, por ejemplo, destinada a alcanzar la igualdad de oportunidades de financiación de los estudios. Desgraciadamente, sólo es posible realizar este último análisis en zonas urbanas.

\section{1. Del lado de la oferta de educación}

Se busca saber si la evolución del vínculo "puro" entre el origen cultural y el éxito escolar depende de los esfuerzos del Estado hacia la educación durante este período. ¿A mayores esfuerzos, mayor igualdad de oportunidades o no existe correlación entre estas dos variables? Una manera de contestar esta pregunta es examinando los coeficientes de correlación entre la variación de variables que aproximan el esfuerzo público en términos de educación -como el gasto de educación o el número de profesores en los establecimientos públicos- y la variación de los parámetros $\beta_{k}$ ( $k$ generaciones) del modelo (2), que expresa la fuerza de este vínculo "puro".

El $\beta_{1}$ — generación nacida entre 1905-1912 en el medio urbano, entre 1913-1922 en el medio rural- se fija en 1. Para las otras generaciones $k$, un $\beta_{k}<1$ significa la distensión del vínculo entre la generación 1 y la generación $k$, si $\beta_{k}>1$, la consolidación. Los datos sobre la oferta de educación que se utilizan aquí son nacionales. Proceden del INEI y fueron recogidos por Portocarrero \& Oliart (1989), con excepción de los datos sobre la enseñanza superior que proceden de la Asamblea Nacional de los Rectores (ANR) (25). El cuadro 10 presenta estos coeficientes de correlación.

\section{Cuadro 10 - Coeficientes de correlación entre la variación de $\beta_{k}$ y la variación de variables de oferta pública en educación.}

\begin{tabular}{|c|c|c|c|}
\hline $\begin{array}{l}\text { Correlación entre la variación del } \beta_{k} \text { del modelo (2) y la de } \\
\ldots\end{array}$ & Lima & Otras ciudades & Rural \\
\hline Proporción del presupuesto gastado en educación & -0.09 & 0.39 & -0.74 \\
\hline Gastos en educación (soles constantes) & -0.10 & 0.35 & -0.62 \\
\hline Número de establecimientos primarios públicos & 0.31 & 0.35 & 0.86 * \\
\hline Número de establecimientos secundarios públicos & 0.01 & $0.91 * * *$ & -0.12 \\
\hline Número de universidades públicas & -0.02 & 0.26 & -0.33 \\
\hline Número de universidades públicas y particulares & 0.04 & 0.10 & -0.36 \\
\hline Número de docentes de primaria pública & 0.67 & $0.99 * * *$ & -0.08 \\
\hline Número de docentes de secundaria pública & -0.33 & $0.95 * *$ & 0.90 * \\
\hline
\end{tabular}

Fuentes: encuestas 1974, 1985-1986, 1990, 1996, 2001 para el cálculo de $\beta_{k}$. Las otras variables se extraen de Portocarrero \& Oliart (1989) y de la Asamblea Nacional de Rectores (ANR) en cuanto al número de universidades.

Notas: ***: coeficiente de correlación significativo al umbral del 1\%; **: al umbral del 5\%; *: al umbral del 10\%. Las variables de oferta de educación están a escala nacional, la distinción Lima/ otras ciudades/ rural se refieren sólo a los coeficientes $\beta_{k}$.

(25) Agradezco a Patricia Arregui que me permitió obtener estos datos. 
Campo: $\operatorname{los} 23$ a 69 años.

Lectura: un signo positivo del coeficiente de correlación significa que la evolución del grado de oportunidad (es decir, de $\beta_{k}$ ) entre dos generaciones se hizo a un mismo ritmo (creciente o decreciente) que la evolución de la variable de oferta de educación — por ejemplo, el aumento del grado de igualdad de oportunidades se hizo más lento al mismo tiempo que el crecimiento del gasto público entre dos generaciones disminuyó-.

Un resultado importante se desprende de este cuadro: en Lima, el esfuerzo del Estado para desarrollar las infraestructuras escolares no ha tenido ningún impacto en la desigualdad de oportunidades — coeficientes de correlación no significativos-. La desigualdad de oportunidades, pues, parece estar vinculada a la demanda de educación, más que a la oferta. En las otras ciudades, en cambio, y en el medio rural en una menor medida, las variaciones del grado de igualdad de oportunidades en el acceso a la educación (26) parecen haber sido correlacionadas con la evolución de la oferta pública de educación. La evolución del grado de oportunidades en las ciudades fuera de Lima ha sido especialmente sensible a variaciones en el ritmo del gasto en la enseñanza secundaria —número de establecimientos y de profesores-y también a las fluctuaciones del ritmo de crecimiento del número de profesores en la enseñanza primaria. Esta doble comprobación puede explicarse de la siguiente manera: en las ciudades fuera de Lima, la desigualdad de oportunidades en el acceso a la educación vinculadas a la oferta son más fuertes cuando se trata de la enseñanza secundaria. La discriminación según el origen social y cultural empieza al inicio de la secundaria. Así, el acceso de los niños a la enseñanza primaria en las ciudades no depende de la cantidad de centros escolares, ya que es probable que este número es — y ha sido — suficientemente importante para satisfacer la demanda de educación primaria — las familias no abandonan la posibilidad de que sus hijos asisten a la primaria por distancias demasiado grandes entre la escuela y su domicilio aun cuando la distancia podría generarles gastos difíciles de asumir-. Por el contrario, el número de profesores de enseñanza primaria tiene una influencia significativa, ya que determina la calidad de la enseñanza primaria que, a su vez, determina el éxito o fracaso en la secundaria. En efecto, un número muy reducido de profesores genera enseñanza primaria de mala calidad tal que puede conducir a la exclusión prematura del sistema escolar de los niños más desfavorecidos por su herencia cultural: estos niños no tienen un entorno familiar que pueda compensar la mala calidad de la enseñanza primaria, ya que los padres no pueden ayudar a sus hijos a aprender lo que se les enseña en la escuela (27). En el medio rural en cambio, aunque la desigualdad

(26) Aunque la variación del grado de oportunidad en las ciudades no tiene trend temporal, tal como se mostró en la parte anterior, esta variación no es despreciable.

(27) En un informe de evaluación acerca del sistema educativo peruano, el Banco Mundial (1999) observa rendimientos educativos desiguales, siendo netamente desfavorecidos los pobres, los pobladores rurales y los indigenas. Los autores del informe sugieren varias políticas para mejorar la equidad en el sistema, tal como incrementar el material de aprendizaje, desarrollar la educación bilingüe, aumentar las compensaciones para docentes quienes van a enseñar en las comunidades e instaurar un sistema que rote a los docentes para que todos vayan a enseñar en las comunidades. También proponen desarrollar programas de nutrición y salud en el seno de la escuela, desarrollar mecanismos de información para los padres a fin de sensibilizarlos acerca de las "virtudes" de la 
de oportunidades parece muy vinculada a la demanda en educación de las familias, se constata que la falta de establecimientos primarios ha desanimado un buen número de familias más desfavorecidas a enviar a sus hijos a la escuela, aumentando así la desigualdad de oportunidades. Así, mientras la educación primaria no ha alcanzado un carácter universal en el medio rural, parece eficaz multiplicar la cantidad de establecimientos primarios públicos con el fin de ganar en igualdad de oportunidades escolares. En las ciudades fuera de la Lima, un medio para disminuir la desigualdad de oportunidades sería mejorando la calidad de la enseñanza primaria, multiplicando el número de profesores e invirtiendo más en la enseñanza secundaria en general.

\section{2. Del lado de la demanda de educación}

La pregunta a la que se busca responder ahora es: ¿Qué factor del origen desempeña el papel más importante en el éxito escolar? ¿El factor socioeconómico o el factor cultural? El origen socioeconómico de una persona se define aquí por la ocupación principal a la que se dedica el padre durante su vida (28). Es efectivamente un buen indicador del nivel de vida del hogar en el momento en que la persona se educó. La información sobre la actividad del padre sólo existe para Lima y las otras ciudades (29), por lo que no se puede estudiar la evolución de la influencia del origen social en el éxito escolar en el medio rural. La metodología utilizada para contestar la pregunta es nuevamente la de los modelos log-lineales. La verificación se construye a partir de cuatro modelos, los modelos (A), (B), (C), (D) que buscan reconstituir la tabla de contingencia formada por las tres siguientes variables: el nivel escolar alcanzado, el origen cultural —nivel de educación alcanzado por el padre-y el origen socioeconómico —ocupación del padre-. Según los modelos, se introducen distintas interacciones entre las variables. La comparación de las cualidades predictivas de cada uno de los modelos permitirá evaluar cuáles son las interacciones más significativas. Los modelos se describen detalladamente en el Anexo 2.

El modelo (A) es muy poco realista. Supone que el nivel escolar alcanzado por una persona es independiente tanto de su origen cultural como socioeconómico. Sólo reconoce la interacción entre el nivel de educación del padre y la ocupación del padre. El papel de este modelo es servir de referencia. El modelo (B), por su parte, considera que, además, de esta correlación entre la educación del padre y su actividad, existe una relación entre el éxito escolar de una persona y su origen cultural. El modelo (C) se diferencia del modelo (B) en que toma en cuenta la dimensión socioeconómica del

escuela, desarrollar la educación pre-primaria en el medio rural en las comunidades y, finalmente, proporcionar educación compensatoria a los niños desfavorecidos.

(28) Codificada en 6 modalidades: 1.Ejecutivo, Profesional, Gerente, Técnico; 2. Administrador, Funcionario; 3. Empleado de oficina; 3. Comerciante; 4. Agricultor, 5. Trabaja en los servicios, transportes; 6. Obrero, Artesano.

(29) En la encuesta de 2001, no hay pregunta sobre la ocupación de los padres. Se dispone solamente, para el medio rural, de la encuesta de 1985-1986 para estudiar la evolución de la influencia de la actividad de los padres en el éxito escolar. El número de personas es entonces insuficiente para realizar un estudio robusto. 
origen en vez de su dimensión cultural. Por último, el modelo (D) es el más completo, puesto que integra tres interacciones. La primera, común a todos los modelos, es la interacción entre la educación del padre y su ocupación, la segunda, la interacción entre el éxito escolar y el origen cultural y la tercera, entre el éxito escolar y el origen socioeconómico. Gracias a estos cuatro modelos, es posible construir dos pruebas con el fin de evaluar la importancia relativa del origen cultural y el origen socioeconómico en la determinación del nivel escolar alcanzado. La primera prueba compara el valor predictivo del modelo (B) y el del modelo (C). La segunda evalúa en el modelo (D) la significatividad de cada una de estas dos interacciones. El cuadro 11 presenta los indicadores de ajuste de los distintos modelos a Lima y en las otras ciudades, distinguiendo hombres y mujeres pero sólo en las otras ciudades, ya que los resultados son los mismos sin distinción de géneros en Lima.

En Lima como en las otras ciudades, el impacto del origen socioeconómico sobre el éxito escolar es significativo, aun cuando se tiene en cuenta el impacto del origen cultural —según el criterio del BIC, el modelo (D) es mejor que el modelo (B) que sólo tiene en cuenta el origen cultural en la explicación del éxito escolar; además, cuando se prueban a partir del modelo (D) los dos impactos por separado, el del origen socioeconómico y el del origen cultural, los dos efectos son significativos-.

Sin embargo, el origen cultural desempeña un papel mucho más importante que el origen socioeconómico - el modelo (B) explica un $83 \%$ de lo que el modelo (A), que no incluye ningún vínculo entre origen y éxito escolar, es incapaz de explicar (30), mientras que el modelo $(\mathrm{C})$ sólo explica un 52\%—; además, el $\chi^{2}$ del efecto del origen cultural es menor que el del origen socioeconómico cuando se verifica la significatividad de cada uno de estos efectos a partir del modelo (D). Pues, la desigualdad de oportunidades escolares se explica más por las desigualdades culturales entre los padres que por las desigualdades socioeconómicas. Este resultado va en contra del modelo de Boudon (1973) según el cual "las diferencias en la calidad de la herencia cultural en función de la clase social sólo explican en una medida muy limitada la desigualdad de oportunidades ante la educación.” (p. 303)

Así, las desigualdades culturales y socioeconómicas entre los grupos sociales se combinan para formar desigualdades en los niveles de educación de los niños. El hecho de que, en zonas urbanas, el origen cultural sea el factor predominante de la influencia familiar que determina el éxito escolar está en acuerdo con la teoría del capital cultural. La consecuencia de esto es que, para disminuir la desigualdad de oportunidades escolares en las ciudades, no es suficiente apoyar económicamente a los hogares pobres para que eduquen más a sus hijos. Reducir, por ejemplo, las desigualdades socioeconómicas con un sistema de becas podría disminuir la desigualdad de oportunidades, pero en menor medida.

(30) Según la estadística $r G^{2}$. 


\section{Cuadro 11 - Indicadores de ajuste de los modelos.}

\begin{tabular}{|c|c|c|c|c|c|c|c|}
\hline Modelos & gdl & $G^{2}$ & $\mathrm{P}$ & $r G^{2}$ & $\Delta \%$ & $\mathrm{BIC}$ & $\chi^{2}$ \\
\hline \multicolumn{8}{|l|}{$\begin{array}{l}\text { Lima (Conjunto) } \\
\text { (A) Sin vínculo entre el origen socio- } \\
\text { económico y el nivel escolar alcanzado }\end{array}$} \\
\hline $\begin{array}{l}\left(E_{p} A_{p}\right)(E) \\
\text { (B) El nivel escolar alcanzado depende del } \\
\text { origen cultural }\end{array}$ & 246 & 5001.6 & 0.000 & 0.0 & 23.5 & 2675.6 & \\
\hline $\begin{array}{l}\left(E_{p} A_{p}\right)\left(E E_{p}\right) \\
\text { (C) El nivel escolar alcanzado depende del } \\
\text { origen socio-económico }\end{array}$ & 210 & 848.2 & 0.000 & 83.0 & 8.3 & -1137.4 & \\
\hline $\begin{array}{l}\left(E_{D} A_{b}\right)\left(E A_{D}\right) \\
\text { (D) El nivel escolar alcanzado depende del } \\
\text { origen cultural y socio-económico }\end{array}$ & 216 & 2416.7 & 0.000 & 51.7 & 14.6 & 374.4 & \\
\hline $\begin{array}{l}\left(E_{p} A_{b}\right)\left(E A_{p}\right)\left(E E_{p}\right) \\
\text { Test del efecto de } X: \text { Modelo (D)-(Modelo(D)-X) }\end{array}$ & 180 & 272.9 & 0.000 & 94.5 & 3.7 & -1429.0 & \\
\hline $\mathrm{X}=\left(\mathrm{EE}_{\mathrm{p}}\right)$ & 30 & & 0.000 & & & & 575.3 \\
\hline $\mathrm{X}=\left(E A_{p}\right)$ & 36 & & 0.000 & & & & 2143.8 \\
\hline \multicolumn{8}{|l|}{$\begin{array}{l}\text { Otras ciudades (Hombres) } \\
\text { (A) Sin vínculo entre el origen socio- } \\
\text { económico y el nivel escolar alcanzado }\end{array}$} \\
\hline $\begin{array}{l}\left(E_{p} A_{b}\right)(E) \\
\text { (B) El nivel escolar alcanzado depende del } \\
\text { origen cultural }\end{array}$ & 246 & 5682.0 & 0.000 & 0.0 & 26.1 & 3366.5 & \\
\hline $\begin{array}{l}\left(E_{b} A_{b}\right)\left(E E_{b}\right) \\
\text { (C) El nivel escolar alcanzado depende del } \\
\text { origen socio-económico }\end{array}$ & 210 & 767.5 & 0.000 & 86.5 & 8.4 & -1209.1 & \\
\hline $\begin{array}{l}\left(E_{D} A_{b}\right)\left(E A_{D}\right) \\
\text { (D) El nivel escolar alcanzado depende del } \\
\text { origen cultural y socio-económico }\end{array}$ & 216 & 3694.2 & 0.000 & 35.0 & 19.6 & 1661.1 & \\
\hline$\left(E_{p} A_{p}\right)\left(E A_{p}\right)\left(E E_{p}\right)$ & 180 & 355.4 & 0.000 & 93.7 & 4.7 & -1338.9 & \\
\hline \multicolumn{8}{|l|}{ Test del efecto de X: Modelo (D)-(Modelo(D)-X) } \\
\hline $\mathrm{X}=\left(\mathrm{EE}_{\mathrm{p}}\right)$ & 30 & & 0.000 & & & & 412.1 \\
\hline$X=\left(E A_{p}\right)$ & 36 & & 0.000 & & & & 3338.8 \\
\hline \multicolumn{8}{|l|}{$\begin{array}{l}\text { Otras ciudades (Mujeres) } \\
\text { (A) Sin vínculo entre el origen socio- } \\
\text { económico y el nivel escolar alcanzado }\end{array}$} \\
\hline $\begin{array}{l}\left(E_{\mathrm{b}} \mathrm{A}_{\mathrm{b}}\right)(\mathrm{E}) \\
\text { (B) El nivel escolar alcanzado depende del } \\
\text { origen cultural }\end{array}$ & 246 & 7233.5 & 0.000 & 0.0 & 27.9 & 4893.9 & \\
\hline $\begin{array}{l}\left(E_{b} A_{b}\right)\left(E E_{b}\right) \\
\text { (C) El nivel escolar alcanzado depende del } \\
\text { origen socio-económico }\end{array}$ & 210 & 749.5 & 0.003 & 89.6 & 8.0 & -1247.7 & \\
\hline $\begin{array}{l}\left(E_{D} A_{b}\right)\left(E A_{D}\right) \\
\text { (D) El nivel escolar alcanzado depende del } \\
\text { origen cultural y socio-económico }\end{array}$ & 216 & 4854.9 & 0.000 & 32.9 & 22.3 & 2800.6 & \\
\hline$\left(E_{p} A_{b}\right)\left(E A_{p}\right)\left(E E_{p}\right)$ & 180 & 324.4 & 0.000 & 95.5 & 4.3 & -1387.5 & \\
\hline \multicolumn{8}{|l|}{ Test del efecto de X: Modelo (D)-(Modelo(D)-X) } \\
\hline $\mathrm{X}=\left(\mathrm{EE}_{\mathrm{p}}\right)$ & 30 & & 0.000 & & & & 425.1 \\
\hline$X=\left(E A_{p}\right)$ & 36 & & 0.000 & & & & 4530.5 \\
\hline
\end{tabular}

Fuentes: encuestas 1974, 1985-1986, 1990, 1996, 2001.

Campo: Las zonas urbanas, los 23 a 69 años. Para el conjunto, el análisis incluye las siete generaciones. Para los hombres y las mujeres considerados separadamente, no se tiene en cuenta la primera generación (muy poca representatividad).

Lectura: la adecuación del modelo a los datos empíricos es medida por la estadística de verosimilitud $G^{2} . G^{2}$ sigue asintoticamente una ley de $\chi^{2}$ a $d d l$ grados de libertad. Si $\mathrm{p}<0,05$, entonces la hipótesis según la cual los datos estimados por el modelo no son significativamente diferentes de los datos empíricos se rechaza al umbral del $5 \% . r G^{2}$ da el porcentaje de la distancia entre 
el modelo (A) y los datos empíricos que explica el modelo en cuestión. $\Delta \%$ es la proporción del número total de personas que debería cambiarse de casilla para que la tabla de datos estimados sea igual a la tabla de datos observados. Ninguno de los modelos estima suficientemente bien los datos como para que la diferencia entre los datos estimados y los datos empíricos sea significativamente nula. Es la razón por la que se introduce el criterio del BIC que tiene en cuenta los tres parámetros $G^{2}, d d l$ y $N$, el número total de personas de la tabla. Este criterio permite afirmar que un modelo debe preferirse al otro si su BIC es más pequeño (al menos de 6 puntos). El indicador del BIC se define así:

$B I C=G^{2}-d d l \ln (N)$

\section{RESUMEN Y CONCLUSIÓN}

Este análisis ha permitido destacar algunos resultados claves para entender el nacimiento de las desigualdades socioeconómicas en el Perú, proporcionando luces sobre la formación de desigualdades en la educación. Demostró cómo las desigualdades en los niveles escolares se han reducido en el siglo pasado, gracias a una muy fuerte expansión del sistema escolar. La educación primaria se ha vuelto casi universal en las ciudades, la distancia entre el nivel escolar de las niñas y el de los niños ha disminuido en las ciudades y también la distancia entre el nivel escolar básico de la población indígena y el de la población no indígena. Sin embargo, la población rural y, en particular, las mujeres del sector rural se beneficiaron mucho menos de dicha expansión que la población de las ciudades. Además, la población indígena permanece discriminada en el acceso a la educación superior y ninguna tendencia hacia una reducción de estas desigualdades se vislumbra.

El vínculo entre el origen cultural y el éxito escolar de una persona se ha debilitado de manera continua en las ciudades. El debilitamiento de este vínculo también se verifica en el sector rural, aunque ha sido más errático. Sin embargo, esta ganancia en términos de movilidad escolar se explica para los hombres, en general, y para las mujeres de las ciudades casi íntegramente por la expansión del sistema escolar. Para los hombres, la desigualdad de oportunidades en la educación escolar se ha mantenido igual. En cambio, las mujeres en el medio rural parecen haber obtenido mayor igualdad de oportunidades a lo largo del siglo pasado.

Varias políticas públicas parecen ser eficaces en aumentar el grado de igualdad de oportunidad escolar. Estas políticas consistirían en mejorar la oferta de educación, procediendo a una mejor distribución geográfica del gasto público en educación, en particular, aumentando el número de centros de educación primaria en el medio rural, invirtiendo, en las ciudades fuera de Lima, en la calidad de la educación primaria y desarrollando la educación secundaria pública. Por último, el Estado puede implementar políticas de apoyo a las familias para financiar los estudios de sus hijos. Pero, si bien estas políticas permitirán aumentar la igualdad de oportunidades escolares, su impacto será limitado por el hecho de que la mayor ventaja/desventaja que puede tener una persona para lograr un cierto nivel escolar procede más de su origen cultural que de su origen socioeconómico.

Una de las limitaciones de este análisis es que no tiene en cuenta la calidad de la educación, por falta de información acerca de este tema. Sin embargo, se ha puesto en 
evidencia que, en el Perú, el desarrollo del sistema educativo se ha acompañado de una disminución de la calidad de la educación, particularmente la educación pública. Un estudio realizado en 1994 por el Laboratorio Latinoamericano de Evaluación de la Calidad de la Educación (LLECE), bajo la dirección de la UNESCO, compara la calidad de la educación primaria en trece países de América Latina (31). Los alumnos de los terceros y cuartos grados de primaria dieron una prueba que evaluara los niveles alcanzados en lenguaje y matemáticas. Los resultados son abrumadores para el Perú. Este ocupa el último lugar de la clasificación (32)en matemáticas y el antepenúltimo lugar en lenguaje. Además, la diferencia de los niveles de los alumnos, sobre todo en lenguaje, entre el medio rural y el medio urbano está entre las más fuertes de los trece países. Al diferenciar a las escuelas públicas de las escuelas particulares, el Perú gana tres puestos en la clasificación de éstas con relación a las escuelas públicas. Pues, los rendimientos escolares son mucho menores en zona rural y en los establecimientos públicos. Otro estudio fue realizado en el Perú en 1998 (33) por el Unidad de Mediación de Calidad Educativa del Ministerio de Educación (34) y consistió en evaluar el nivel escolar de alumnos de primaria y secundaria en zonas urbanas. Este estudio pone en evidencia que, en los departamentos más pobres, los rendimientos escolares son los más bajos. En este sentido, si todos los niños peruanos tienen acceso a la educación básica - en las ciudades, por lo menos_- queda claro que no tienen acceso a la misma educación. Los rendimientos de la educación básica son mucho más bajos en zonas rurales que en zonas urbanas, en los departamentos menos desarrollados socioeconómicamente que en los más desarrollados y en los centros escolares públicos que en los particulares.

La disminución de la calidad de la educación pública hace que se subestima mucho la desigualdad de oportunidades escolares, ya que crea una segmentación adicional de la población en función de las posibilidades de acceder a una educación de calidad. La expansión de la educación ha hecho que, en la mayoría de los casos, los grupos desfavorecidos se acerquen a los grupos más favorecidos en su acceso a la educación. Una vez que el límite máximo del desarrollo de la cobertura educativa se alcance, tal como es el caso para la educación primaria en medio urbano, la diferenciación entre los grupos se basará a partir de la calidad de la educación.

Anteriormente, el reto de las políticas públicas en educación era permitir a todos el acceso a la educación. Es necesario añadir un segundo reto, el de lograr que todos tengan acceso a una educación de calidad.

(31) Argentina, Bolivia, Brasil, Chile, Colombia, Costa Rica, Cuba, Honduras, México, Paraguay, Perú, la República Dominicana y Venezuela (Ministerio de Educación, 2001).

(32) Para el tercer grado de primaria. 2000).

(33) CRECER (Crecer con Calidad y Equidad en el Rendimiento) (Ministerio de Educación,

(34) La muestra está constituida por 576 escuelas primarias y 566 escuelas secundarias. 


\section{Referencias citadas}

ANDERSEN, L.E., 2001a - Social Mobility in Latin America: Links with Adolescent Schooling, 39p.; Banco Interamericano de Desarrollo, Working Paper 433.

ANDERSEN, L.E., 2001b - Low Social Mobility in Bolivia: Causes and Consequences for Development, 47p.; Kiel Institute of World Economics, Working Paper 1046.

ANSION, J., LAZARTE, A., MATOS, S., RODRIGUEZ, J. \& VEGA-CENTENO, P., 1998 Educación: La Mejor Herencia, Decisiones educativas y expectativas de los padres de familia, una aproximación empírica, 277p.; Lima: Fondo editorial de la Pontifica Universidad Católica del Perú.

ASAMBLEA NACIONAL DE RECTORES (ANR) - http://www.anr.edu.pe

BANCO MUNDIAL, 1999 - Peru: Education at a Crossroads. Challenges and Opportunities for the 21 st Century, 203p.; Work Bank Report 19006666-PE.

BARTHOLOMEW, D., 1982 -Stochastic models for social processes, 377p.; New York: Wiley.

BECKER, G. \& TOMES, N., 1986 - Human Capital and the Rise and Fall of Families. Journal of Labor Economics, 4(3): S1-S39.

BEHRMAN, J.R., BIRDSALL, N. \& SZEKELY, M., 1998 - Intergenerational Schooling Mobility and Macro Conditions and Schooling Policies in Latin America, 42p., Banco Interamericano de Desarrollo, Working Paper 386.

BEHRMAN, J.R., BIRDSALL, N. \& SZEKELY, M., 2001 - Intergenerational Mobility in Latin America, 37p.; Banco Interamericano de Desarrollo, Working Paper 452.

BINDER, M. \& WOODRUFF, C., 2002 - Inequality and Intergenerational Mobility in Schooling: The case of Mexico. Economic Development and Cultural Change, 50: 249-267.

BISHOP, Y.M.M., FIENBERG, S.E. \& HOLLAND, P.W., 1975 - Discrete Multivariate Analysis: Theory and Practice, 559p.; Cambridge: MIT Press.

BLOSSFELD, H-P. \& SHAVIT, Y., 1993 - Persistent Inequality: Changing Educational Attainment in Thirteen Countries, 395p.; Colorado: Westview Press.

BOUDON, R., 1973 - L'inégalité des chances (3ème édition 1979), 398p.; Paris: Armand Colin. BOURDIEU, P. \& PASSERON, J.-C., 1964 - Les héritiers, 192p.; Paris: Éditions de minuit.

BOURDIEU, P. \& PASSERON, J.-C., 1970 - La reproduction, 284p.; Paris: Éditions de minuit.

BOURGUIGNON, F., FERREIRA, F. \& MENENDEZ, M., 2001 - Inequality of Outcomes, Inequality of Opportunities and Intergenerational Education Mobility in Brazil, 45p.; mimeo.

CHECCHI, D., ICHINO, A. \& RUSTICHINI, A., 1999-More equal but less mobile? Education financing and intergenerational mobility in Italy and in the US. Journal of Public Economics, 74: 351-393.

DAHAN, M. \& GAVIRIA, A., 1999 - Sibling Correlations and Social Mobility in Latin America, 29p., Banco Interamericano de Desarrollo, Working Paper 395.

ERIKSON, R. \& GOLDTHORPE, J.H., 1992 - The Constant Flux. A Study of Class Mobility in Industrial Societies, 429p.; Oxford: Clarenton Press.

FIELDS, G. \& OK, E., 1999 - The measurement of income mobility: an introduction to the literature. In: Handbook on Income Inequality Measurement (ed: Jacques Silber): 557598; KLUWER Academic.

GANZEBOOM, H. \& LUIJKX, R., 2001 - Recent trends in intergenerational occupational class mobility: men in the Netherland 1970-1999,27p.; Mannheim Meeting Research Comitee on Social Stratification (RC28-ISA).

HEATH, A. \& PAYNE, C., 1999 - Twentieth century trend in social mobility in Britain, 35p.; CREST, Working Paper 70. 
HERRERA, J., 2002 - La Pobreza en el Perú 2001: una visión departamental, 196p.; INEI-IRD.

MERTON, R. 1953 - Reference group theory and social mobility. In: Class, Status and Power (Bendix R., Lipset S.M. eds); New York: Free Press.

MINISTERIO DE EDUCACIÓN, 2000 - Resultados de las pruebas de matemática y lenguaje, ¿Qué aprendimos a partir de la Evaluación CRECER 1998. Crecer Boletín, 5/6: 1-11.

MINISTERIO DE EDUCACIÓN, 2001 - El Perú en el primer estudio internacional comparativo de la UNESCO sobre lenguaje, matemática y factores asociados en tercer y cuarto grado. Boletín UMC, 9: 1-19.

MINISTERIO DE TRABAJO Y PROMOCIÓN SOCIAL, 1967 - Encuesta de Hogares de Lima Metropolitana, Enero-Marzo.

PORTOCARRERO, G., OLIART, P., 1989 - El Perú desde la escuela, 236p.; Lima: Instituto de apoyo agrario

ROEMER, J.E., 1998 - Equality of opportunity, 120p., London: Harvard University Press.

SAAVEDRA, J \& MELZI, R., 1998 - Financiamiento de la educación pública en el Perú. In: Financiamiento de la educación pública América Latina:212-267; Santiago: ed. PREALUNESCO.

SAAVEDRA, J. \& SUAREZ, P., 2002 - El financiamiento de la educación pública en el Perú: el rol de las familias, 125p.; Lima: Grade, Documento de trabajo 38.

SHORROCKS, A., 1978 - The measurement of mobility. Econometrica, 46(5): 1013-1024.

THEIL, H., 1972 - Statistical decomposition analysis, 337p.; Amsterdam: North Holland Pub. Co.

THELOT, C. \& VALLET, L.-A., 2000 - La réduction des inégalités sociales devant l'école depuis le début du siècle, Économie et Statistiques, 334: 3-32.

VANDEGAER,D., MARTINEZ, M.\& SCHOKKAERT,E., 1998-Measuring intergenerational mobility and equality of opportunity, 26p.; mimeo.

XIE, Y., 1992 - The Log-multiplicative Layer Effect Model for Comparing Mobility Tables. American Sociological Review, 57: 380-395. 


\section{ANEXO 1: LOS ÍNDICES DE MOVILIDAD CONSTRUIDOS A PARTIR DEL ENFOQUE AXIOMÁTICO}

Shorrocks (1978) propone tres axiomas, (I), (MP) y (M) que debería comprobar un índice de movilidad:

Sea una matriz de transición P, cuya suma de cada fila es igual a 1.

(I) Inmovilidad

$\forall P \in \Gamma \quad M[P] \geq M[I]$

(MP) Movilidad perfecta

Sea $\quad P^{M}=\frac{1}{n} u^{\prime} \quad$ con $\quad u=(1, \ldots, 1)^{\prime} . \quad$ En $\quad$ tal $\quad$ caso $\quad \forall P \in \Gamma \neq P^{M}, \quad M\left[P^{M}\right]>M[P]$

(M) Monotonía

Si $\quad p_{i j}^{\prime} \geq p_{i j} \quad \forall i \neq j \quad y \quad \exists p_{i j}^{\prime}>p_{i j}$, en tal caso $M\left[P^{\prime}\right]>M[P]$

Los dos primeros axiomas asignan límites a los índices. El primer axioma (I) pone la matriz de transición de inmovilidad perfecta como la matriz identidad. El índice de inmovilidad tiene el más bajo valor cuando la matriz de transición da a cada persona una probabilidad nula de alcanzar un destino $j$ diferente de su origen $i$. El segundo axioma (MP) asocia el índice más alto a la matriz de transición de movilidad perfecta, es decir, la matriz por la cual las personas de origen $i$ tienen la misma probabilidad de alcanzar cada uno de los destinos $j$. Entonces, un índice que comprueba el axioma (MP) permite evaluar la movilidad en términos de independencia del estado final respecto al estado inicial (1) o también como medio para igualar las oportunidades. Por último, por el tercer axioma (M), una matriz de transición P' cuyos elementos diagonales son más bajos que los de la matriz $\mathrm{P}$, tendrá un índice de movilidad más alto que la matriz P. Este axioma traduce entonces una

(1) Si se considera la movilidad en términos de independencia del estado final con respecto al estado inicial, la matriz de transición de una sociedad perfectamente móvil es:

$$
A=\left[\begin{array}{ll}
1 / 2 & 1 / 2 \\
1 / 2 & 1 / 2
\end{array}\right] .
$$

Todos los individuos tienen la misma probabilidad de alcanzar un estado dado, cualquiera que sea su estado inicial. Este concepto de la movilidad es el más cercano al de igualdad de oportunidades. 
concepción de la movilidad en términos de movimiento (2). El axioma (M) implica el axioma (I), pero los axiomas (M) y (MP) son incompatibles (3). Por ejemplo, un índice que comprueba $(\mathrm{M})$ determinará la matriz A como más móvil que la $\mathrm{B}$, mientras que un índice que verifica el axioma (MP) juzgue la A menos móvil que la B. Se elegirán entonces los índices de movilidad de modo que comprueben los axiomas (I) y (MP). Van de Gaer et al. (1998) proponen dos axiomas adicionales de modo que el índice de movilidad traduzca el grado de igualdad de oportunidades de la sociedad en cuestión. Estos axiomas son:

(ANT) Anonimato entre los tipos

Sea $E[P]$ una matriz obtenida a partir de la matriz $\mathrm{P}$ permutando las filas de $\mathrm{P}$. En tal caso, $M[E[P]=M[P]$.

(DEOT) Atractivo de las transformaciones que igualan las oportunidades

Si $\widetilde{P}$ puede obtenerse por una secuencia terminada de EOT, en tal caso, $M[\widetilde{P}]=M(P)$, donde EOT es una transformación que iguala las oportunidades, definida como

$$
\begin{array}{lll}
\widetilde{p}_{i j}=p_{i j}-\varepsilon ; & \widetilde{p}_{i k}=p_{i k}+\varepsilon ; \quad \widetilde{p}_{l j}=p_{l j}+\varepsilon ; \quad \widetilde{p}_{l k}=p_{l k}-\varepsilon ; \quad \varepsilon>0, \quad j<k, \\
\widetilde{C}_{i, r} \geq \widetilde{C}_{l, r} \quad \forall r=1, \ldots, n \quad \text { en } \quad \text { donde } \quad \widetilde{C}_{l, r}=\sum_{j=1}^{r} \widetilde{p}_{l, j}
\end{array}
$$

El axioma (ANT) es incompatible con el axioma (M) puesto que, por ejemplo, un índice que comprueba el axioma (M) será el mismo para la matriz de perfecta inmovilidad - matriz identidad - y la de perfecta movilidad como generadora de movimientos.

Se proponen otros axiomas en la literatura económica y sociológica. No se trata de hacer aquí una revisión exhaustiva. A menudo, se definen índices que no son válidos para el presente caso, ya sea porque miden la movilidad de una variable cuantitativa continua como el ingreso, ya sea porque sólo tienen un sentido bajo ciertas condiciones que no se pueden aceptar en el presente caso — matrices monótonas — o ya sea porque se refieren a un aspecto de la movilidad que no es el más pertinente aquí.

Los índices de movilidad que se presentan aquí son índices que aproximan la movilidad como medio para aumentar la independencia del estado futuro con respecto al estado de origen. Se construye un grupo de índices a partir de los valores propios de la matriz de transición. El valor propio más elevado de la matriz de transición $P$ después

(2) Si se concibe la movilidad en términos de movimiento, entonces la matriz de transición de una sociedad perfectamente móvil es:

$$
B=\left[\begin{array}{ll}
0 & 1 \\
1 & 0
\end{array}\right] \text {, es decir que todos los individuos intercambian }
$$

su posición. En la sociedad caracterizada por la matriz de transición B, los individuos realizan más movimientos que en la sociedad caracterizada por la A, pero su situación final depende enteramente de su situación inicial.

(3) Para una justificación, referirse a Shorrocks (1978). 
de $\lambda_{1}$ (igual a 1), $\lambda_{2}$, indica la velocidad —o más bien su opuesto- a la cual la matriz $P$ permite, en un proceso de Markov, pasar de la distribución inicial $\pi_{0}$ a la distribución del equilibrio estable $\pi_{e}$, es decir, el vector para el cual $\pi_{e}^{\prime}=\pi_{e}^{\prime} P$. Más precisamente, la velocidad asintotica de convergencia es igual a $-\log \left|\lambda_{2}\right|$. Ahora bien, la relación entre la velocidad de convergencia y la movilidad es muy estrecha (Theil, 1972). Si una sociedad es perfectamente móvil, la matriz $P$ permite alcanzar el vector de equilibrio en un único período. Es el caso en que $\left|\lambda_{2}\right|=0$. Al contrario, cuanto más rígida es una sociedad o, mejor dicho, cuanto más baja es la movilidad social, más la velocidad de convergencia es débil. Los índices construidos a partir de $\lambda_{2}$ son los siguientes:

$$
M[P]=1-\left|\lambda_{2}\right|
$$

y el índice de Shorrocks:

$$
M_{h}[P]=\exp \left[\frac{\log 2}{\log \left|\lambda_{2}\right|}\right]
$$

Estos dos índices se encuentran entre 0 (inmovilidad perfecta) y 1 (movilidad perfecta). Comprueban los axiomas de inmovilidad (I) y de movilidad perfecta (MP), pero no el de monotonía (M).

Se construyen otros dos índices a partir de los valores propios de la matriz, pero no comprueban el axioma de movilidad perfecta (MP): el índice de Prais y el índice construido a partir de la media geométrica de los valores propios.

En el caso de una inmovilidad perfecta $P=I$ y, por lo tanto, todos los valores propios son iguales a uno. Pues, la media aritmética de los valores propios puede ser una medida de la movilidad. Ahora bien, por definición, la suma de los valores propios es igual a la traza de la matriz. De ahí el índice de Prais:

$$
M_{t}[P]=\frac{n-\operatorname{Tr}[P]}{n-1} \text { en donde } \operatorname{Tr}[P] \text { es la traza de la matriz } P \text { y } n \text { es la dimensión }
$$

de la matriz de transición.

$M_{t}[P]$ verifica los axiomas de inmovilidad (I) y de monotonía (M), pero no el de movilidad perfecta (MP).

La crítica que se puede formular en cuanto a este índice es que reduce mucho la información contenida en la matriz de transición, ya que no tiene en cuenta los elementos de la matriz fuera de la diagonal principal.

De la misma manera, el valor absoluto de la media geométrica de los valores propios proporciona cierta medida de la movilidad. Supongamos $n$, la dimensión de la matriz cuadrada $P$. Puesto que el producto de los valores propios de la matriz $P$ es igual a su determinante, se define el índice $M_{d}[P]$ del siguiente modo:

$$
M_{d}[P]=1-|P|^{1 /(n-1)}
$$

Este índice sólo satisface el axioma de inmovilidad (I). 
Un índice que comprueba no sólo los axiomas (I) y (MP) sino también el axioma (ANT) es el que mide la distancia entre $P$ y el vector de equilibrio definido por $\pi^{\prime}=\pi^{\prime} P^{\text {: }}$

$M_{f}[P]=1-\frac{1}{n^{2}} \sum_{i=1}^{n} \sum_{j=1}^{n}\left|\frac{p_{i j}-\pi_{j}}{\pi_{j}}\right|$

dónde $\pi_{j}$ es el elemento $j$ del vector de equilibrio $\pi$.

Existen numerosos otros índices. pero sólo tienen en cuenta el aspecto "movimiento" de la movilidad, tal como el índice de Bartholomew (1982). No se los tendrá en cuenta, pues no es relevante este el aspecto de la movilidad en el presente estudio.

\section{ANEXO 2: PRESENTACIÓN DE LOS MODELOS LOG- LINEALES}

\section{PRINCIPIO DEL MÉTODO (4)}

El principio de la estimación por los modelos log-lineales es reconstituir una tabla de contingencia de distintas variables introduciendo interacciones entre las variables y, luego, probar si la tabla de contingencia teórica así obtenida es significativamente similar a la tabla de contingencia real. Por ejemplo, si la educación de una persona es independiente de la de su padre, sería posible reconstituir la tabla de contingencia con dos dimensiones, conformada por estas dos variables, poniendo que cada célula es igual al producto de los márgenes de la fila y columna que corresponden a la célula. El modelo se escribiría en forma logarítmica así:

$\ln \left(F_{i, k}\right)=\mu+\lambda_{i}^{O}+\lambda_{k}^{E}$

en donde $F_{i, k}$ es la frecuencia estimada de la célula $(i, k), O$ el nivel de educación del padre, $E$ la educación de la persona, $\lambda_{i}^{O}, \lambda_{k}^{E}$ los logaritmos de los márgenes de la fila $i$ y de la columna $k$ respectivamente. Si la independencia se comprueba, una prueba de chi-2 pone de manifiesto que la frecuencia estimada es significativamente igual a la frecuencia real. Si no es el caso, se introduce un término de interacción entre la educación de la persona y la de su padre $\lambda_{i, k}^{O E}$, que es el logaritmo de la relación entre la verdadera frecuencia de la célula y la frecuencia que tendría si las dos variables fueran independientes. En tal caso el logaritmo de la frecuencia tiene una estimación perfecta en la siguiente relación:

$\ln \left(F_{i, j, k}\right)=\mu+\lambda_{i}^{O}+\lambda_{k}^{E}+\lambda_{i, k}^{O E}$. Es el modelo saturado.

Si $\lambda_{i, k}^{O E}=0$, las dos variables son independientes. lineales.

(4) Referirse a Bishop et al. (1975) para una descripción más formal de los modelos log- 


\section{PRESENTACIÓN DE LOS MODELOS}

\section{Modelo (1)}

El modelo (1) supone tres interacciones, una entre la educación del padre y la generación, otra entre la educación de la persona y la generación y, finalmente, la tercera entre la educación de la persona y la de su padre. Este modelo se llama a menudo modelo de fluidez social constante (CnSF: constant social fluidity). Se define así:

$$
\ln \left(F_{i, j, k}\right)=\mu+\lambda_{i}^{O}+\lambda_{j}^{G}+\lambda_{k}^{E}+\lambda_{i, j}^{O G}+\lambda_{k, j}^{E G}+\lambda_{i, k}^{O E}
$$

en donde $\mathrm{O}$ designa el origen social, desde el nivel de educación del padre (7 modalidades), E la educación de la persona (7 modalidades) y G la generación ( 7 modalidades para Lima y las otras ciudades, 6 para el medio rural); $F_{i, j, k}$ la frecuencia de la célula $(i, j, k)$ de la tabla de tres entradas $(O, E, G) ; \lambda_{i}^{O}, \lambda_{k}^{E}, \lambda_{j}^{G}$ son los efectos principales de $O, E, G$ sobre la distribución de los individuos respectivamente y, finalmente, $\lambda_{i, j}^{O G}, \lambda_{k, j}^{E G}, \lambda_{i, k}^{O E}$ son las interacciones parciales entre $O$ y $G$ dado $E, E$ y $G$ dado $O$ y, finalmente, $O$ y $E$ dado $G$, respectivamente.

\section{Modelo (2)}

El modelo (2) es un modelo de tipo UniDiff (5), es decir que sin ser un modelo saturado introduce una triple interacción entre la educación de la persona, su origen social y su generación. Verifica pues, además de las interacciones del modelo (1), el efecto del tiempo, es decir, de la generación, sobre el vínculo entre la educación de la persona y la de su padre. Se define así:

$$
\ln \left(F_{i, j, k}\right)=\mu+\lambda_{i}^{O}+\lambda_{j}^{G}+\lambda_{k}^{E}+\lambda_{i, j}^{O G}+\lambda_{k, j}^{E G}+\lambda_{i, k}^{O E}+\beta_{j} \psi_{i, k}
$$

en donde $\psi_{i, k}$ traduce la forma de la asociación estadística entre la educación de la persona y la de su padre y $\beta_{j}$ es un parámetro que expresa, para la generación $j$, la fuerza relativa de esta asociación. Si para la primera generación el coeficiente $\beta$ está fijado en $1\left(\beta_{1}=1\right)$, un parámetro inferior a 1 para otra generación $j$ significa que el vínculo "puro" entre el origen cultural y el éxito escolar se distendió entre la primera generación y la generación $j$. Al contrario, si $\beta_{j}>1$, el vínculo es más fuerte o en otros términos, la fluidez es más baja. El modelo se dice uniforme, ya que supone que todos los "odds ratios" — las relaciones de las probabilidades relativas — varían en el mismo sentido y con la misma intensidad $\beta_{j}$ para la generación $j$ : si el vínculo se relaja, se relaja con la misma fuerza entre todas las categorías.

\section{Modelo (A): no hay vínculo entre origen social y éxito escolar}

En el modelo (A), se tiene en cuenta una sola interacción, la existente entre la educación del padre y la actividad del padre. El modelo (A), modelo de referencia, se define del siguiente modo:

(5) Modelo log-multiplicativo "Uniform Difference", introducido en la literatura por Erikson \& Goldth (1992); Xie (1992). 


$$
\ln \left(F_{i, j, k}\right)=\mu+\lambda_{i}^{E}+\lambda_{j}^{E_{p}}+\lambda_{k}^{A_{p}}+\lambda_{j, k}^{E_{p} A_{p}}
$$

en donde $E$ designa la educación de la persona ( 7 modalidades), $E_{p}$ su origen cultural aproximado desde el nivel de educación de su padre ( 7 modalidades), $A_{p}$ su origen socioeconómico aproximado desde la actividad de su padre (6 modalidades); $F_{i, j, k}$ la frecuencia de la célula $(i, j, k)$ de la tabla de tres entradas $\left(E, E_{p}, A_{p}\right) ; \lambda_{i}^{E}, \lambda_{j}^{E_{p}}, \lambda_{k}^{A_{p}}$ son los efectos principales de $E, E_{p}, A_{p}$ respectivamente sobre la distribución de los individuos y, finalmente, $\lambda_{j, k}^{E_{p} A_{p}}$ es la interacción parcial entre $E_{p}$ y $A_{p}$ dado $E$.

\section{Modelo (B): el éxito escolar depende del origen cultural}

El modelo (B) contiene, además de las interacciones del modelo (A), una interacción entre la educación de la persona y la de su padre:

$$
\ln \left(F_{i, j, k}\right)=\mu+\lambda_{i}^{E}+\lambda_{j}^{E_{p}}+\lambda_{k}^{A_{p}}+\lambda_{j, k}^{E_{p} A_{p}}+\lambda_{i, j}^{E E_{p}}
$$

\section{Modelo (C): el éxito escolar depende del origen socioeconómico}

El modelo $(\mathrm{C})$ es idéntico al modelo $(\mathrm{A})$ con, además, la interacción entre la actividad del padre y la educación de la persona interrogada:

$$
\ln \left(F_{i, j, k}\right)=\mu+\lambda_{i}^{E}+\lambda_{j}^{E_{p}}+\lambda_{k}^{A_{p}}+\lambda_{j, k}^{E_{p} A_{p}}+\lambda_{i, j}^{E A_{p}}
$$

\section{Modelo (D): el éxito escolar depende del origen social y el origen cultural}

El modelo (D) incluye tres interacciones, una entre la educación del padre y la actividad del padre, otra entre el origen socioeconómico y el éxito escolar y, finalmente, la tercera entre el origen cultural y el éxito escolar:

$$
\ln \left(F_{i, j, k}\right)=\mu+\lambda_{i}^{E}+\lambda_{j}^{E_{p}}+\lambda_{k}^{A_{p}}+\lambda_{j, k}^{E_{p} A_{p}}+\lambda_{j, k}^{E A_{p}}+\lambda_{i, j}^{E E_{p}}
$$

